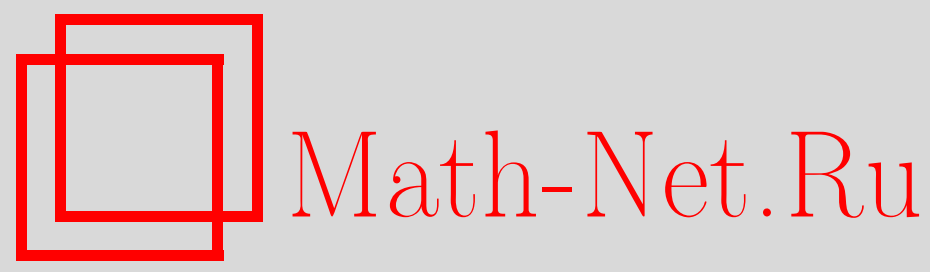

И. П. Чухров, О соотношении тупиковых и минимальных комплексов граней в единичном кубе, Дискрет. матем., 2012, том 24, выпуск 2, 46-74

DOI: https://doi.org/10.4213/dm1183

Использование Общероссийского математического портала Math-Net.Ru подразумевает, что вы прочитали и согласны с пользовательским соглашением http://www.mathnet.ru/rus/agreement

Параметры загрузки:

IP : 54.157 .27 .8

26 апреля 2023 г., 13:40:33 


\title{
О соотношении тупиковых и минимальных комплексов граней в единичном кубе
}

\author{
() 2011 г. И. П. Чухров
}

\begin{abstract}
Рассматривается задача о соотношении тупиковых и минимальных дизъюнктивных нормальных форм или комплексов граней булевой функции, которая была поставлена С. В. Яблонским в связи с оцениванием сложности алгоритмов минимизации булевых функций. В работе исследуются комплексы граней, минимальные относительно класса мер сложности, для которых сложность комплекса уменьшается при удалении грани и не изменяется при замене некоторых граней на грани изоморфные относительно перестановки координат.

Для отношения числа тупиковых и минимальных комплексов граней функции для любой меры сложности из такого класса получена нижняя оценка логарифма вида $c n 2^{n}$, где $c>1,355 \cdot 2^{-7}$. При этом логарифм числа тупиковых комплексов может быть порядка $n 2^{n}$ при единственном минимальном комплексе граней, если мера сложности удовлетворяет дополнительному условию: сложность комплекса уменьшается при уменьшении ранга любой грани.

Получены нижние оценки мощности классов функций, по порядку логарифма сравнимые с числом всех функций, для которых отношение числа тупиковых и минимальных комплексов, в том числе при условии единственного минимального комплекса, имеют порядок роста логарифма, равный $n 2^{n}$.
\end{abstract}

\section{1. Введение}

Характерной чертой задачи минимизации булевых функций в классе дизъюнктивных нормальных форм (далее д.н.ф.) (см. $[1,2,3])$, то есть построения для функции ее минимальной д.н.ф. относительно некоторой меры сложности, является возможность нахождения оптимального решения путем применения простых локальных упрощений: уменьшение ранга и удаление импликанты д.н.ф. Понятие сложности базируется на естественном представлении, что при уменьшении ранга или удалении импликанты сложность д.н.ф. не увеличивается. Число импликант и сумма рангов импликант в д.н.ф. являются простейшими мерами сложности.

Полиэкстремальность задачи минимизации булевой функции заключается в возможности существования большого числа локальных экстремумов, в роли которых выступают тупиковые д.н.ф., среди которых содержатся глобальные экстремумы - минимальные д.н.ф. При этом произвольная д.н.ф. может быть преобразована в эквивалентную тупиковую локальным алгоритмом 2-го порядка [1].

Эффективное решение задачи минимизации возможно для специальных классов функций, а именно, в тех случаях, когда известны структура и сложность минимальной д.н.ф., 
например, для монотонных, линейных и других ядровых функций, для цепных, циклических и симметрических функций. В общем случае, известные алгоритмы минимизации функций в качестве одного из этапов содержат перебор по определенной стратегии некоторого множества д.н.ф. и, в конечном итоге, гарантированно строят тупиковую д.н.ф. Естественно возникает вопрос о возможности получения в результате выполнения алгоритма минимальной д.н.ф. При этом отношение числа минимальных и тупиковых д.н.ф. является оценкой вероятности нахождения глобального экстремума, то есть минимальной д.н.ф.

Задача минимизации булевых функций обычно рассматривается в двух эквивалентных моделях - аналитической и геометрической. В аналитической модели используются понятия булевой функции, импликанты, д.н.ф. и т.д., зависящие от $n$ переменных. В геометрической модели эквивалентными понятиями являются подмножества вершин, грани, комплексы граней и т.д., в $n$-мерном единичном кубе (см. с. 307 в [4]). В дальнейшем изложении будем использовать понятия и обозначения, связанные с гранями и множествами вершин единичного куба $B^{n}$, в следующей геометрической интерпретации.

Гранью единичного куба $B^{n}$ называется множество вершин

$$
B_{i_{1}, \ldots, i_{k}}^{n, \sigma_{1}, \ldots, \sigma_{k}}=\left\{\tilde{x}=\left(x_{1}, \ldots, x_{n}\right) \in B^{n} \mid x_{i_{1}}=\sigma_{1}, \ldots, x_{i_{k}}=\sigma_{k}\right\}
$$

где $\sigma_{s} \in\{0,1\}$ для $s=1, \ldots, k$. Множество индексов $\left\{i_{1}, \ldots, i_{k}\right\}$ называется направлением грани, число $k$ называется рангом, и число $n-k$ называется размерностью грани. Множество всех вершин куба $B^{n}$ является гранью ранга 0. Грань может быть представлена в виде $\left\{\tilde{x} \in B^{n} \mid \tilde{\alpha} \leqslant \tilde{x} \leqslant \tilde{\beta}\right\}$, где $\tilde{\alpha}$ и $\tilde{\beta}-$ минимальная и максимальная вершины грани, при этом расстояние Хемминга $\rho(\tilde{\alpha}, \tilde{\beta})$ равно размерности грани. В таком представлении множество вершин грани называется интервалом размерности $k$.

Комплекс граней

$$
M=\left\{I_{r}, r=1, \ldots, l\right\}
$$

покрывает множество вершин

$$
N_{M}=\bigcup_{r=1}^{l} I_{r} \subseteq B^{n} .
$$

Комплексу граней $M$ однозначно соответствует функция $f \in P_{n}$, для которой $N_{M}=N_{f}$, где $N_{f}$ - множество единичных вершин функции в кубе $B^{n}$, то есть множество наборов значений переменных, на которых функция равна 1. Реализация булевой функции $f$ в виде д.н.ф. эквивалентна покрытию гранями подмножества вершин $N_{f} \subset B^{n}$. Любой комплекс граней $M$, для которого $N_{M}=N_{f}$, называется комплексом граней функции $f$.

Два комплекса граней называются эквивалентными, если они покрывают одно подмножество вершин единичного куба $B^{n}$, то есть являются комплексами граней одной функции.

Для множества вершин $Q \subseteq B^{n}$ любая грань $I \subseteq Q$ называется допустимой гранью. Допустимая грань $I$ для множества вершин $Q \subseteq B^{n}$ называется максимальной, если не существует такой грани $I^{\prime}$, что $I \subset I^{\prime} \subseteq Q$.

Комплекс граней $M$ называется неприводимым, если после удаления из него любой грани получается комплекс граней не эквивалентный $M$. В неприводимом комплексе $M$ каждая грань $I \in M$ содержит хотя бы одну вершину, которая не покрывается другими гранями из комплекса $M$. Такая вершина называется собственной вершиной грани $I$ в неприводимом комплексе. 
Комплекс граней $M$ называется тупиковым, если он является неприводимым и все грани в нем являются максимальными для множества $N_{M}$.

Грань $I$ называется ядровой для множества вершин $Q \subset B^{n}$, если она является максимальной для множества $Q$ и существует вершина $\tilde{\alpha} \in I$, которая не принадлежит никакой другой грани, максимальной для $Q$. Вершины ядровой грани $I$, которые не покрываются никакими другими максимальными для множества $Q$ гранями, называются собственными вершинами ядровой грани $I$. Вершины ядровой грани, которые не являются собственными для ядровой грани, называются регулярными.

Комплекс граней $M$ в кубе $B^{n}$ называется ядровым, если любая грань комплекса $M$ является ядровой для подмножества вершин $N_{M} \subset B^{n}$.

Переход к геометрической модели позволяет абстрагироваться от конкретного набора переменных д.н.ф. и перейти к размерности, то есть к числу переменных, и нумерации координат единичного куба. Это позволяет упростить понятие изоморфизма для комплексов граней по сравнению с д.н.ф., используемое при определении меры сложности.

Обозначим через $\pi^{n}$ множество перестановок координат в единичном кубе $B^{n}$. Для перестановки $\pi \in \pi^{n}$ обозначим через $\pi(I)$ грань, которая получается из грани $I$ перестановкой координат $\pi$, то есть

$$
\pi\left(B_{i_{1}, \ldots, i_{k}}^{n, \sigma_{1}, \ldots, \sigma_{k}}\right)=B_{\pi\left(i_{1}\right), \ldots, \pi\left(i_{k}\right)}^{n, \sigma_{1}, \ldots, \sigma_{k}},
$$

и через

$$
\pi(M)=\left\{\pi\left(I_{r}\right), r=1, \ldots, l\right\}
$$

обозначим комплекс граней, который получается из комплекса $M=\left\{I_{r}, r=1, \ldots, l\right\}$ перестановкой координат $\pi$ во всех гранях комплекса.

Комплексы граней называются изоморфными в кубе $B^{n}$, если один комплекс может быть получен из другого комплекса перестановкой координат, то есть для комплексов граней $M_{1}$ и $M_{2}$ существует перестановка координат $\pi \in \pi^{n}$ такая, что

$$
M_{1}=\pi\left(M_{2}\right) \text {. }
$$

При этом грани являются изоморфными только тогда, когда они имеют одинаковую размерность и одинаковое число координат направления, для которых в вершинах грани значение равно 0, и, следовательно, одинаковое число координат направления, для которых в вершинах грани значение равно 1.

Д.н.ф. называются изоморфными, если одна д.н.ф. может быть получена из другой подстановкой переменных без отождествлений. При этом импликанты являются изоморфными только тогда, когда в них одинаковое число переменных без отрицания и с отрицанием, соответственно они имеют одинаковый ранг и размерность.

Изоморфизм аналитической и геометрической моделей основан на изоморфизме элементарных конъюнкций и граней единичного куба $B^{n}$. Элементарная конъюнкция $K=x_{i_{1}}^{\sigma_{1}} \cdots x_{i_{r}}^{\sigma_{r}}$ изоморфна грани $B_{K}^{n}=B_{i_{1}, \ldots, i_{r}}^{n, \sigma_{1}, \ldots, \sigma_{r}}$, д.н.ф. $K_{1} \vee \ldots \vee K_{s}$ изоморфна комплексу граней $\left\{B_{K_{j}}^{n}, j=1, \ldots, s\right\}$.

Определение 1. Функционал $\mathscr{L}$, определенный на множестве всех комплексов граней (д.н.ф.), является мерой сложности, если он удовлетворяет следующим аксиомам (см. с. 298 в [4]).

(1) Аксиома неотрицательности: $\mathscr{L}(M) \geqslant 0$ для любого комплекса $M$. 
(2) Аксиома монотонности: $\mathscr{L}(M \cup\{I\}) \geqslant \mathscr{L}\left(M \cup\left\{I^{\prime}\right\}\right)$ для любого комплекса $M$ и любой грани $I$ при условии $I \subset I^{\prime}$.

(3) Аксиома выпуклости: $\mathscr{L}\left(M_{1} \cup M_{2}\right) \geqslant \mathscr{L}\left(M_{1}\right)+\mathscr{L}\left(M_{2}\right)$ для любого представления комплекса $M=M_{1} \cup M_{2}$ в виде прямого объединения комплексов $M_{1}$ и $M_{2}$, то есть не имеющих общих членов.

(4) Аксиома инвариантности относительно изоморфизма: $\mathscr{L}(M)=\mathscr{L}(\pi(M))$ для любого комплекса $M$ и любой перестановки координат $\pi$.

Примерами мер сложности могут служить следующие функционалы (в терминах д.н.ф.): $\mathscr{L}_{V}-$ число дизъюнкций, $\mathscr{L}_{\&}-$ число конъюнкций, или $\mathscr{L}_{\neg}-$ число отрицаний в д.н.ф. Мера сложности, равная числу граней в комплексе $M$, то есть число импликант в д.н.ф. (функционал $\left.\mathscr{L}_{V}+1\right)$, называется длиной и обозначается $l(M)$. Мера сложности, равная сумме рангов граней в комплексе $M$, то есть число переменных в д.н.ф. (функционал $\left.\mathscr{L}_{V}+\mathscr{L}_{\&}+1\right)$, называется сложностью и обозначается $L(M)$. Мера сложности, которая соответствует функционалу $\alpha \mathscr{L}_{\&}+\beta \mathscr{L}_{\vee}$, где $\alpha+\beta>0, \alpha \geqslant 0, \beta \geqslant 0$, называется линейной сложностью и обозначается $L_{\alpha, \beta}(M)$.

Определение 2. Комплекс граней называется $\mathscr{L}$-минимальным, если он имеет наименьшую меру сложности $\mathscr{L}$ среди всех эквивалентных комплексов граней. $\mathscr{L}$-минимальному комплексу граней изоморфна $\mathscr{L}$-минимальная д.н.ф. Кратчайшим называется $l$-минимальный комплекс, и минимальным называется $L$-минимальный комплекс.

Будем использовать следующие обозначения подмножеств вершин $B^{n}$ :

$B_{k}^{n}=\left\{\tilde{x} \in B^{n} \mid \rho(\tilde{0}, \tilde{x})=\|\tilde{x}\|=k\right\}$ есть $k$-й слой куба $B^{n}$;

$S_{m-k, m}^{n}=\left\{\tilde{x} \in B^{n} \mid m-k \leqslant\|\tilde{x}\| \leqslant m\right\}$ есть пояс в кубе $B^{n}$, состоящий из слоев с номерами $m-k, \ldots, m$, где $0 \leqslant k \leqslant m \leqslant n$.

Функция называется симметрической, если она не меняет значения при любой перестановке переменных (см. [2]). Функция называется поясковой, если множество единичных вершин функции в кубе $B^{n}$ совпадает с поясом. Симметрическая функция однозначно представляется в виде набора поясковых функций, которые являются компонентами связности (см. [2]) симметрической функции.

Используемые, но не определяемые в этой статье понятия и определения можно найти в $[1,2,4]$. Через $\lfloor x\rfloor$ (соответственно $\lceil x\rceil$ ) будем обозначать целую часть (соответственно верхнюю целую часть) числа $x$. Под $\log$ всюду понимается логарифм по основанию 2 . Асимптотические оценки числовых параметров в единичном кубе $B^{n}$ всюду получаются при $n \rightarrow \infty$.

Для функции $f \in P_{n}$ будем обозначать

$\mathscr{T}(f)$ множество тупиковых комплексов граней функции;

$M_{\mathscr{L}}(f)$ множество $\mathscr{L}$-минимальных комплексов граней функции;

$\tau(f)$ число тупиковых комплексов граней функции;

$\mu_{\mathscr{L}}(f)$ число $\mathscr{L}$-минимальных комплексов граней функции;

$\chi_{\mathscr{L}}(f)=\tau(f) / \mu_{\mathscr{L}}(f)$ отношение числа тупиковых и $\mathscr{L}$-минимальных комплексов граней функции. 
Максимальные значения параметров $\tau(f), \mu_{\mathscr{L}}(f)$ и $\chi_{\mathscr{L}}(f)$ на множестве функций $P_{n}$ будем обозначать $\tau(n), \mu_{\mathscr{L}}(n)$ и $\chi_{\mathscr{L}}(n)$. Будем использовать обозначения без указания меры сложности, например, $\chi(f)$ или $\chi(n)$ в утверждениях, которые справедливы одновременно для минимальных и кратчайших тупиковых комплексов.

Верхняя оценка для $\chi(n)$ является следствием очевидного соотношения $\chi(n) \leqslant \tau(n)$ и оценки числа тупиковых д.н.ф. функции (см. с. 110 в [2]):

$$
\log \chi(n) \leqslant \log \tau(n) \lesssim n 2^{n} \log (3 / 2)
$$

Нижние оценки для отношения числа тупиковых и минимальных д.н.ф. булевой функции последовательно улучшались в работах ряда авторов. Ю. И. Журавлев в [5] построил пример функции

$$
f\left(x_{1}, \ldots, x_{n}\right)=S_{1,2}^{3}\left(x_{1}, x_{2}, x_{3}\right)\left(x_{4} \oplus \ldots \oplus x_{n}\right),
$$

для которой

$$
\tau(f)=5^{2^{n-4}}, \quad \mu(f)=2^{2^{n-4}},
$$

так как

$$
\tau\left(S_{1,2}^{3}\right)=5, \quad \mu\left(S_{1,2}^{3}\right)=2,
$$

то есть при $n>4$

$$
\chi(n) \geqslant \chi(f)=2^{2^{n-4} \log (5 / 2)} .
$$

Следующая нижняя оценка $\chi(n)$ явилась следствием результатов о числе минимальных и тупиковых д.н.ф. для почти всех функций $f \in P_{n}$ при $n \rightarrow \infty$. В. В. Глаголевым в [6] приведены оценки для почти всех функций длин сокращенной д.н.ф.

$$
s(f)=2^{n} n^{\log \log n(1+o(1))}
$$

и кратчайшей д.н.ф.

$$
\frac{2^{n-1}}{\log n \log \log n} \lesssim l(f) \lesssim \frac{2^{n-1}}{\log } \log n \log n,
$$

из которых следует следующая верхняя оценка числа минимальных д.н.ф. для почти всех функций:

$$
\log \mu(f) \leqslant \log \sum_{l \leqslant l(f)}\left(\begin{array}{c}
s(f) \\
l
\end{array}\right) \lesssim l(f) \log \frac{s(f)}{l(f)} \lesssim 2^{n-1}(\log \log n)^{2}
$$

А. А. Сапоженко в [7] показал, что

$$
\log \tau(f) \sim \log n \log \log n 2^{n-1}
$$

для почти всех функций. Так как

$$
\log \mu(f)=o(\log \tau(f)), \quad \log \chi(f) \sim \log \tau(f)
$$


для почти всех функций, справедлива оценка

$$
\log \chi(n) \gtrsim 2^{n-1} \log n \log \log n .
$$

Неполная определенность булевых функций (см. с. 81 в [1]) позволяет существенно упростить конструирование функций с экстремальными значениями характеристик. В [8] была построена не всюду определенная функция $f$ с единственной минимальной д.н.ф., такая, что

$$
\log \chi(f)=\log \tau(f) \gtrsim 2^{n-1} \sqrt{n / 2 \pi} .
$$

В $[9,10]$ для максимального значения $\chi\left(S_{n}\right)$ параметра $\chi(f)$ на классе симметрических функций $S_{n} \subset P_{n}$ была получена оценка

$$
\log \chi(n) \geqslant \log \chi\left(S_{n}\right) \geqslant c n\left(\begin{array}{c}
n \\
\lfloor n / 2\rfloor
\end{array}\right) \asymp \sqrt{n} 2^{n} .
$$

Улучшить оценку $\chi(n)$ удалось после повышения нижней оценки $\tau(n)$. В [11] было показано, что при достаточно больших $n$ справедливы оценки

$$
\log \tau(n) \geqslant c n 2^{n}, \quad \log \chi(n) \geqslant c n 2^{n-2},
$$

где $c>0,75 \cdot 2^{-5}$, то есть

$$
\log \chi(n) \asymp n 2^{n}
$$

при $n \rightarrow \infty$.

Получение нетривиальных нижних оценок $\chi_{\mathscr{L}}(n)$ связано с решением двух задач: обоснованием свойств $\mathscr{L}$-минимальных комплексов и наличием тупиковых не $\mathscr{L}$-минимальных комплексов граней для функции.

Если $\mathscr{L}$-минимальный комплекс $M$ не является неприводимым, то есть $M=M_{1} \cup M_{0}$, где комплекс $M_{1}$ эквивалентен $M$, то $\mathscr{L}\left(M_{0}\right)=0$. Действительно, в силу аксиом неотрицательности и выпуклости

$$
\mathscr{L}(M)=\mathscr{L}\left(M_{1} \cup M_{0}\right) \geqslant \mathscr{L}\left(M_{1}\right)+\mathscr{L}\left(M_{0}\right) \geqslant 0 .
$$

Так как комплексы $M_{1}$ и $M$ являются эквивалентными и $\mathscr{L}$-минимальными комплексами, то $\mathscr{L}(M)=\mathscr{L}\left(M_{1}\right)$ и, следовательно, $\mathscr{L}\left(M_{0}\right)=0$.

Любой комплекс граней, имеющий нулевую меру $\mathscr{L}$-сложности, является $\mathscr{L}$-минимальным в силу аксиомы неотрицательности. Все комплексы, которые могут быть получены из комплекса нулевой $\mathscr{L}$-сложности уменьшением ранга граней, удалением граней и перестановкой координат во всех гранях, также имеют нулевую $\mathscr{L}$-сложность, то есть являются $\mathscr{L}$-минимальными. Например, любой комплекс граней, в котором все грани содержат

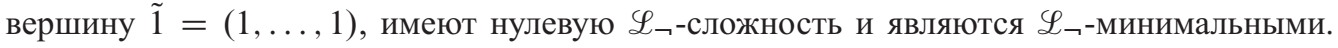
Таким образом, предположение, что $\mathscr{L}$-минимальный комплекс $M$ может не являться неприводимым, приводит к существованию и необходимости изучения множества комплексов нулевой $\mathscr{L}$-сложности.

С другой стороны, из любого $\mathscr{L}$-минимального комплекса уменьшением ранга и удалением граней можно получить эквивалентный тупиковый $\mathscr{L}$-минимальный комплекс функции. Следовательно, для любой функции $f$ справедливы соотношения

$$
M_{\mathscr{L}}(f) \cap T(f) \neq \varnothing
$$


И

$$
\frac{|\mathcal{T}(f)|}{\left|M_{\mathscr{L}}(f) \cap \mathscr{T}(f)\right|} \geqslant \frac{\tau(f)}{\mu_{\mathscr{L}}(f)}=\chi_{\mathscr{L}}(f),
$$

то есть значение $1 / \chi_{\mathscr{L}}(f)$ является верхней оценкой вероятности получить тупиковый $\mathscr{L}$-минимальный комплекс функции $f$, и оценивание параметра $\chi_{\mathscr{L}}(f)$ имеет смысл не только для мер сложности, относительно которых любой $\mathscr{L}$-минимальный комплекс является тупиковым.

Все комплексы граней из множества $\mathscr{T}(f) \cap M_{\mathscr{L}}(f)$ имеют одинаковую и минимальную $\mathscr{L}$-сложность, а комплексы из множества $\mathscr{T}(f) \backslash \mathcal{M}_{\mathscr{L}}(f)$ имеют большую сложность, чем $\mathscr{L}$-минимальные комплексы. Поэтому для получения оценки $\chi_{\mathscr{L}}(f)>1$ необходимо построение функции $f$, у которой существует не менее двух неизоморфных множеств тупиковых комплексов - минимальные и не минимальные тупиковые комплексы граней.

Для сравнения сложности комплексов граней будем использовать свойства, а не значение функционала меры сложности. Определим классы мер сложности, обладающие дополнительными к аксиомам свойствами, которые однозначно устанавливают отношение эквивалентности или строго предпочтения по сложности для сравниваемых комплексов граней.

Определение 3. Мера сложности $\mathscr{L}$ удовлетворяет свойству строгой монотонности относительно длины, если $\mathscr{L}$-сложность комплекса уменьшается при удалении грани, которая не содержит вершин $\tilde{0}$ и $\tilde{1}$.

Условие, что $\mathscr{L}$-сложность комплекса уменьшается только при удалении грани, которая не содержит вершин $\tilde{0}$ и $\tilde{1}$, позволяет рассматривать меры сложности, для которых могут существовать не тупиковые комплексы нулевой сложности, например, для меры

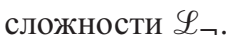

Определение 4. Мера сложности $\mathscr{L}$ удовлетворяет свойству строгой монотонности относительно сложности, если $\mathscr{L}$-сложность комплекса уменьшается при уменьшении ранга или удалении произвольной грани.

Определение 5. Мера сложности $\mathscr{L}$ удовлетворяет усиленному свойству инвариантности относительно изоморфизма, если при замене в комплексе произвольного набора граней на изоморфные грани сложность комплекса не изменяется.

Введем следующие обозначения для классов мер сложности:

$\Lambda_{l}$ есть класс мер сложности, которые удовлетворяют свойству строгой монотонности относительно длины;

$\Lambda_{L}$ есть класс мер сложности, которые удовлетворяют свойству строгой монотонности относительно сложности;

$\Lambda_{\pi}$ есть класс мер сложности, которые удовлетворяют усиленному свойству инвариантности относительно изоморфизма.

Очевидно, что $\Lambda_{L} \subset \Lambda_{l}$ и для любой меры сложности $\mathscr{L} \in \Lambda_{L}$ все $\mathscr{L}$-минимальные комплексы являются тупиковыми. При этом отметим, что используемые при минимизации функционалы являются мерами сложности из классов $\Lambda_{\pi} \cap \Lambda_{l}$ или $\Lambda_{\pi} \cap \Lambda_{L}$.

В статье предлагаются методы построения булевых функций, которые позволяют получить высокие нижние оценки отношения числа тупиковых и минимальных комплексов граней для мер сложности из классов $\Lambda_{\pi} \cap \Lambda_{l}$ и $\Lambda_{\pi} \cap \Lambda_{L}$. Методы основаны на развитии 
результатов $[11,12]$ и используют свойства специальных тупиковых комплексов граней в единичном кубе размерности, меньшей $n$, для определения функции $n$ переменных.

Для логарифма отношения числа тупиковых и минимальных комплексов граней функции для всех мер сложности из класса $\Lambda_{\pi} \cap \Lambda_{l}$ получена нижняя оценка вида $c n 2^{n}$, где $c>1,355 \cdot 2^{-7}$, при достаточно больших $n$. При этом число тупиковых комплексов, по порядку роста логарифма равное $n 2^{n}$, может быть при единственном минимальном комплексе граней для всех мер сложности из класса $\Lambda_{\pi} \cap \Lambda_{L}$.

Получены нижние оценки мощности классов функций $P_{n}$, для которых число тупиковых комплексов, а также, при условии существования единственного минимального комплекса для всех мер сложности из $\Lambda_{\pi} \cap \Lambda_{L}$, отношение числа тупиковых и минимальных комплексов для всех мер сложности из $\Lambda_{\pi} \cap \Lambda_{l}$ имеют порядок роста логарифма, равный $n 2^{n}$. Показано, что мощности этих классов функций по порядку логарифма равны $2^{n}$, то есть сравнимы с числом всех функций $P_{n}$.

Статья имеет следующую структуру.

В разделе 2 приводится описание методов построения функций, обладающих экстремальным соотношением числа тупиковых и минимальных комплексов граней в единичном кубе.

В разделе 3 приводятся формулировки основных результатов о соотношении тупиковых и минимальных комплексов граней в единичном кубе.

В разделе 4 приводятся доказательства лемм и теорем.

\section{2. Описание конструкции}

Определим следующие параметры для комплексов граней функции $f \in P_{n}$ :

$\chi \mathscr{L}(f)=\tau(f) / \mu_{\mathscr{L}}(f)$ есть отношение числа тупиковых и $\mathscr{L}$-минимальных комплексов функции;

$\chi_{\mathbf{C}}(f)=\min _{\mathscr{L} \in \mathbf{C}} \chi_{\mathscr{L}}(f)$ есть минимальное отношение числа тупиковых и $\mathscr{L}$-минимальных комплексов функции для класса мер сложности $\mathbf{C}$;

$\tau\left(f \mid \mu_{\mathscr{L}}(f)=1\right)$ есть число тупиковых комплексов функции при условии существования единственного $\mathscr{L}$-минимального комплекса;

$\tau\left(f \mid \mu_{\mathbf{C}}(f)=1\right)$ есть число тупиковых комплексов функции при условии $\mu_{\mathscr{L}}(f)=1$ для любой меры сложности $\mathscr{L}$ из класса мер сложности С.

Обозначим через $\chi_{\mathscr{L}}(n), \chi_{\mathbf{C}}(n), \tau\left(n \mid \mu_{\mathscr{L}}=1\right)$ и $\tau\left(n \mid \mu_{\mathbf{C}}=1\right)$ максимальные значения этих параметров на множестве функций $P_{n}$.

В единичном кубе $B^{n}$ введем следующие обозначения:

$\varphi^{n}$ есть множество всех граней;

$\mathscr{T}^{n}$ есть множество всех тупиковых комплексов граней;

$M_{\mathscr{L}}^{n}$ есть множество $\mathscr{L}$-минимальных комплексов граней;

$\mathscr{T}_{m-k, m}^{n}$ есть множество тупиковых комплексов $k$-мерных граней в поясе $S_{m-k, m}^{n} \subset B^{n}$, где $0<k<m<n$; 
$\mathscr{F}_{m-k, m}^{n}$ есть множество функций из $P_{n}$, для которых все максимальные грани являются $k$-мерными гранями в поясе $S_{m-k, m}^{n}$ и, соответственно, $\mathscr{T}(f) \subseteq \mathscr{T}_{m-k, m}^{n}$, где $0<k<m<n$.

$C_{M}$ есть подмножество собственных вершин граней неприводимого, тупикового или ядрового комплекса $M$, которое содержит по одной произвольной, если их несколько, собственной вершине для каждой грани комплекса.

Лемма 1. Для любого класса мер сложности $\mathbf{C}$ и любой меры сложности $\mathscr{L} \in \mathbf{C}$ справедливы оценки

$$
\begin{gathered}
\chi_{\mathbf{C}}(n) \leqslant \chi_{\mathscr{L}}(n) \leqslant \tau(n), \\
\tau\left(n \mid \mu_{\mathbf{C}}=1\right) \leqslant \tau\left(n \mid \mu_{\mathscr{L}}=1\right) \leqslant \tau(n) .
\end{gathered}
$$

Для числа тупиковых комплексов функции справедлива оценка

$$
\log \tau(n)<n 2^{n} \log (3 / 2)+2^{n} \log e .
$$

Нижняя оценка $\chi_{\Lambda_{\pi} \cap \Lambda_{l}}(n)$ основана на построении специальной функции $n$ переменных по функции $f \in \underset{m-k, m}{\mathscr{F}_{m-2}}$, где $0<k<m<\lfloor(n-2) / 2\rfloor$. Определим функцию $\Phi_{f} \in P_{n}$ следующим образом:

$$
\Phi_{f}\left(x_{1}, \ldots, x_{n}\right)=\Phi_{f, 0,0}\left(x_{1}, \ldots, x_{n}\right) \vee \Phi_{f, 0,1}\left(x_{1}, \ldots, x_{n}\right) \vee \Phi_{f, 1,1}\left(x_{1}, \ldots, x_{n}\right),
$$

где

$$
\begin{aligned}
\Phi_{f, 0,1}\left(x_{1}, \ldots, x_{n}\right)= & \bar{x}_{n-1} \cdot x_{n} \cdot S_{m-k, m}^{n-2}\left(x_{1}, \ldots, x_{n-2}\right), \\
\Phi_{f, 0,0}\left(x_{1}, \ldots, x_{n}\right)= & \bar{x}_{n-1} \cdot \bar{x}_{n} \cdot S_{m-k, m}^{n-2}\left(x_{1}, \ldots, x_{n-2}\right) \cdot \bar{f}\left(x_{1}, \ldots, x_{n-2}\right) \\
& \cdot\left(x_{1} \oplus \ldots \oplus x_{n-2} \oplus 1\right), \\
\Phi_{f, 1,1}\left(x_{1}, \ldots, x_{n}\right)= & x_{n-1} \cdot x_{n} \cdot S_{m-k, m}^{n-2}\left(x_{1}, \ldots, x_{n-2}\right) \cdot \bar{f}\left(x_{1}, \ldots, x_{n-2}\right) \\
& \quad \cdot\left(x_{1} \oplus \ldots \oplus x_{n-2}\right)
\end{aligned}
$$

и $S_{m-k, m}^{n-2}\left(x_{1}, \ldots, x_{n-2}\right)$ есть поясковая функция в кубе $B^{n-2}$.

Для функции $\Phi_{f}\left(x_{1}, \ldots, x_{n}\right)$ введем следующие подмножества вершин куба $B^{n}$ (см. рис. 1):

$$
\begin{aligned}
S_{\Phi_{f}} & =\left\{\tilde{x} \in B^{n} \mid \bar{x}_{n-1} \cdot x_{n} \cdot S_{m-k, m}^{n-2}\left(x_{1}, \ldots, x_{n-2}\right)=1\right\} \subset B_{\bar{x}_{n-1} x_{n}}^{n}, \\
H_{f} & =\left\{\tilde{x} \in B^{n} \mid \bar{x}_{n-1} \cdot x_{n} \cdot f\left(x_{1}, \ldots, x_{n-2}\right)=1\right\} \subseteq S_{\Phi_{f}} \subset B_{\bar{x}_{n-1} x_{n}^{n}}, \\
R_{\Phi_{f}} & =S_{\Phi_{f}} \backslash H_{f} \subset B_{\bar{x}_{n-1} x_{n}}^{n}, \\
C_{\Phi_{f}} & =C_{\Phi_{f}, 0} \cup C_{\Phi_{f}, 1},
\end{aligned}
$$

где

$$
C_{\Phi_{f}, 0}=\left\{\tilde{x} \in B^{n} \mid \Phi_{f, 0,0}\left(x_{1}, \ldots, x_{n}\right)=1\right\} \subset B_{\bar{x}_{n-1} \bar{x}_{n}}^{n}
$$

И

$$
C_{\Phi_{f}, 1}=\left\{\tilde{x} \in B^{n} \mid \Phi_{f, 1,1}\left(x_{1}, \ldots, x_{n}\right)=1\right\} \subset B_{x_{n-1} x_{n}}^{n} .
$$




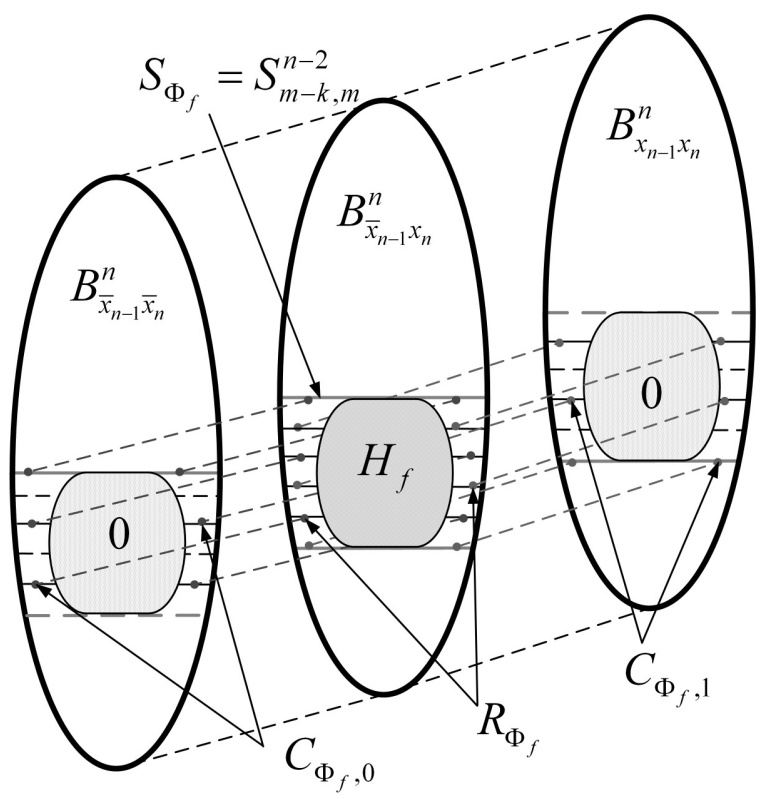

Рис. 1. Определение функции $\Phi_{f}$

Множество вершин $C_{\Phi_{f}}$ принадлежит либо четным слоям грани $B_{\bar{x}_{n-1}}^{n} \bar{x}_{n}$, либо нечетным слоям грани $B_{x_{n-1} x_{n}}^{n}$, так как является подмножеством вершин линейной функции. Для каждой вершины множества $C_{\Phi_{f}}$ существует единственная максимальная грань, содержащая эту вершину, которая является одномерной ядровой гранью функции $\Phi_{f}$. Соответственно, каждая вершина из множества $C_{\Phi_{f}}$ является собственной вершиной одномерной ядровой грани, при этом вторая вершина в ядровой грани принадлежит множеству $R_{\Phi_{f}}$. Поэтому допустимой гранью функции $\Phi_{f}$, содержащей вершину из множества $C_{\Phi_{f}}$, может быть либо 0-мерная грань, совпадающая с вершиной, либо одномерная ядровая грань.

Обозначим через $\operatorname{Ker}\left(\Phi_{f}\right)$ комплекс граней, который состоит из всех ядровых одномерных граней функции $\Phi_{f}$ с собственными вершинами из множества $C_{\Phi_{f}}$. Вершины из множества $R_{\Phi_{f}}$ являются регулярными для ядровых граней из комплекса $\operatorname{Ker}\left(\Phi_{f}\right)$.

В грани $B_{\bar{x}_{n-1} x_{n}}^{n} \subset B^{n}$ множество $S_{\Phi_{f}}$ совпадает с поясом $S_{m-k, m}^{n-2}$, для которого максимальными гранями являются $k$-мерные грани. При этом любая допустимая грань функции $\Phi_{f}$, которая содержит вершину из множества $H_{f}$, принадлежит множеству $S_{\Phi_{f}}$.

Отметим, что допустимые грани функции $\Phi_{f}$ не содержат вершин 0 и $\tilde{1}$, поэтому сложность комплекса граней функции $\Phi_{f}$ строго уменьшается при удалении любой грани комплекса для любой меры сложности из $\Lambda_{\pi} \cap \Lambda_{l}$.

Определение 6. Прямым объединением множеств комплексов граней $\mathscr{G}_{1}$ и $\mathscr{G}_{2}$ в кубе $B^{n}$, для которых не существует граней, одновременно принадлежащих комплексам из множеств $\mathscr{G}_{1}$ и $\mathscr{G}_{2}$, называется множество комплексов

$$
\mathscr{G}_{1} \cup \mathscr{G}_{2}=\left\{M \mid M=M_{1} \cup M_{2}, M_{1} \in \mathscr{G}_{1}, M_{2} \in \mathscr{G}_{2}\right\} .
$$


Введем обозначения для следующих множеств комплексов граней:

$\varphi^{n}\left(C_{\Phi_{f}}\right)$ есть множество комплексов граней, в которые входит одна допустимая грань функции $\Phi_{f}$ для каждой вершины из множества $C_{\Phi_{f}}$;

$\varphi^{n}\left(S_{\Phi_{f}}\right)$ есть множество комплексов граней, в которые входит не более $b_{m}^{n-2}=\left(\begin{array}{c}n-2 \\ m\end{array}\right)$ допустимых граней для множества $S_{\Phi_{f}}$;

$\Omega^{n}\left(\Phi_{f}\right)=\varphi^{n}\left(S_{\Phi_{f}}\right) \cup \varphi^{n}\left(C_{\Phi_{f}}\right)$.

Справедливы следующие утверждения.

Лемма 2. Для функции $f \in \underset{m-k, m}{\mathscr{F}_{m}^{n-2}}$ и любой мерь сложности $\mathscr{L} \in \Lambda_{\pi} \cap \Lambda_{l}$, где $0<k<m \leqslant\lfloor(n-2) / 2\rfloor$, справедливо включение

$$
M_{\mathscr{L}}\left(\Phi_{f}\right) \subseteq \Omega^{n}\left(\Phi_{f}\right) .
$$

Лемма 3. Для функиии $f \in \underset{m-k, m}{\mathscr{F}_{m-2}^{n-2}}$ и любой меры сложности $\mathscr{L} \in \Lambda_{\pi} \cap \Lambda_{l}$, где $0<k<m \leqslant\lfloor(n-2) / 2\rfloor$ справедливы неравенства

$$
\tau\left(\Phi_{f}\right) \geqslant \tau(f), \quad \mu_{\mathscr{L}}\left(\Phi_{f}\right) \leqslant 2^{c \sqrt{n} 2^{n}},
$$

где с - положительная постоянная.

Отображение $\mathscr{A}: B^{n} \rightarrow B^{n}$, где $\mathscr{A}\left(x_{1}, \ldots, x_{n}\right)=\left(\bar{x}_{1}, \ldots, \bar{x}_{n}\right)$ определяет автоморфизм единичного куба $B^{n}$, при котором сохраняется бинарное отношение смежности вершин в единичном кубе. Соответственно, сохраняются свойства принадлежности вершины подмножеству (грани, слою, поясу и т.д.), максимальности грани для некоторого подмножества вершин, неприводимости комплекса граней и другие.

Для слоя $B_{m}^{n}$ образом является слой $\mathscr{A}\left(B_{m}^{n}\right)=B_{n-m}^{n}$, а для пояса $S_{m-k, m}^{n}$ образом является пояс $\mathscr{A}\left(S_{m-k, m}^{n}\right)=S_{n-m, n-m+k}^{n}$, где $0 \leqslant k \leqslant m \leqslant n$.

Для функций $f \in P_{n}$, то есть подмножеств вершин $N_{f}$ в единичном кубе $B^{n}$, автоморфизм $\mathscr{A}$ определяется отображением $\mathscr{A}: P_{n} \rightarrow P_{n}$, где $f_{\mathscr{A}}\left(x_{1}, \ldots, x_{n}\right)=f\left(\bar{x}_{1}, \ldots, \bar{x}_{n}\right)$. Соответственно, образом множества функций $\mathscr{F}_{m-k, m}^{n}$ является множество функций $\mathscr{A}\left(\mathscr{F}_{m-k, m}^{n}\right)=\mathscr{F}_{n-m, n-m+k}^{n}$.

Следовательно, утверждения, справедливые для тупиковых комплексов множества $\mathscr{T}_{m-k, m}^{n}$ и функций из $\mathscr{F}_{m-k, m}^{n}$, являются справедливыми и для изоморфных им тупиковых комплексов множества $\mathscr{T}_{n-m, n-m+k}^{n}$ и функций из $\mathscr{F}_{n-m, n-m+k}^{n}$.

Для функции $f \in \mathscr{F}_{m-k, m}^{n-2}$, где $0<k<m \leqslant\lfloor(n-2) / 2\rfloor$, определим функцию $\Phi_{f}^{*} \in P_{n}$ следующим образом:

$$
\Phi_{f}^{*}\left(x_{1}, \ldots, x_{n}\right)=\Phi_{f, 0,0}^{*}\left(x_{1}, \ldots, x_{n}\right) \vee \Phi_{f, 0,1}^{*}\left(x_{1}, \ldots, x_{n}\right) \vee \Phi_{f, 1,1}^{*}\left(x_{1}, \ldots, x_{n}\right),
$$

где

$$
\begin{gathered}
\Phi_{f, 0,1}^{*}\left(x_{1}, \ldots, x_{n}\right)=\bar{x}_{n-1} \cdot x_{n} \cdot S_{n-2-m, n-2-m+k}^{n-2}\left(x_{1}, \ldots, x_{n-2}\right), \\
\Phi_{f, 0,0}^{*}\left(x_{1}, \ldots, x_{n}\right)=\bar{x}_{n-1} \cdot \bar{x}_{n} \cdot S_{n-2-m, n-2-m+k}^{n-2}\left(x_{1}, \ldots, x_{n-2}\right) \\
\cdot \bar{f}\left(\bar{x}_{1}, \ldots, \bar{x}_{n-2}\right) \cdot\left(x_{1} \oplus \ldots \oplus x_{n-2} \oplus 1\right), \\
\Phi_{f, 1,1}^{*}\left(x_{1}, \ldots, x_{n}\right)=x_{n-1} \cdot x_{n} \cdot S_{n-2-m, n-2-m+k}^{n-2}\left(x_{1}, \ldots, x_{n-2}\right) \\
\cdot \bar{f}\left(\bar{x}_{1}, \ldots, \bar{x}_{n-2}\right) \cdot\left(x_{1} \oplus \ldots \oplus x_{n-2}\right)
\end{gathered}
$$


и $S_{n-2-m, n-2-m+k}^{n-2}\left(x_{1}, \ldots, x_{n-2}\right)$ - поясковая функция в кубе $B^{n-2}$.

Утверждения, аналогичные леммам 2 и 3 , справедливы для функции $\Phi_{f}^{*} \in P_{n}$, определяемой по функции $f \in \underset{m-k, m}{\mathscr{F}_{m}^{n-2}}$, то есть по функции $f\left(\bar{x}_{1}, \ldots, \bar{x}_{n-2}\right) \in \mathscr{F}_{n-2-m, n-2-m+k}^{n-2}$.

Заметим, что в комплексы граней из $g^{n}\left(S_{\Phi_{f}}^{*}\right)$ входит не более $b_{n-2-m}^{n-2}=\left(\begin{array}{c}n-2 \\ m\end{array}\right)$ допустимых граней для множества $S_{\Phi_{f}}^{*}$.

Очевидно, что при $m<\lfloor(n-2) / 2\rfloor$ множества вершин функций $\Phi_{f}$ и $\Phi_{f}^{*}$ являются несвязанными компонентами в кубе $B^{n}$, так как множество вершин $N_{\Phi_{f}}$ находится ниже слоя с номером $\lfloor n / 2\rfloor$, а множество вершин $N_{\Phi_{f}^{*}}$ находится выше слоя с номером $\lfloor n / 2\rfloor$ в кубе $B^{n}$.

Тупиковые, кратчайшие и минимальные комплексы обладают свойством суммируемости (см. с. 117 в [2]) для компонент связности: комплекс граней, который получается прямым объединением любых тупиковых, соответственно, кратчайших или минимальных комплексов граней для компонент связности, является тупиковым, соответственно, кратчайшим или минимальным комплексом.

Для произвольных мер сложности утверждение о суммируемости $\mathscr{L}$-минимальных комплексов для компонент связности в общем случае неверно, но лемму 1 можно обобщить следующим образом.

Лемма 4. Включение

$$
M_{\mathscr{L}}\left(\Phi_{f} \vee \Phi_{f}^{*}\right) \subseteq \Omega^{n}\left(\Phi_{f}\right) \cup \Omega^{n}\left(\Phi_{f}^{*}\right)
$$

справедливо при $f \in \underset{m-k, m}{\mathscr{F}_{m}^{n-2}} u \mathscr{L} \in \Lambda_{\pi} \cap \Lambda_{l}$, где $0<k<m<\lfloor(n-2) / 2\rfloor$.

Известно (см. [12]), что существует функция $f \in \mathscr{F}_{m-k, m}^{n}$, где $m=\lfloor n / 2\rfloor-1$ и $k=\lfloor 0,1052 m\rfloor$, для которой

$$
\log \tau(f) \gtrsim c_{\tau} n 2^{n-1},
$$

где $c_{\tau}>1,355 \cdot 2^{-5}$. Поэтому из лемм 2-4 следует, что для некоторой функции $f \in \underset{m-k, m}{\mathscr{F}^{n}} \stackrel{n-2}{ }$, где $m=\lfloor(n-2) / 2\rfloor-1$, справедливы оценки

$$
\begin{aligned}
\log \tau\left(\Phi_{f} \vee \Phi_{f}^{*}\right) & \gtrsim 2 c_{\tau}(n-2) 2^{n-3}, \\
\log \mu_{\mathscr{L}}\left(\Phi_{f} \vee \Phi_{f}^{*}\right) & =O\left(\sqrt{n} 2^{n}\right)
\end{aligned}
$$

для любой меры сложности $\mathscr{L} \in \Lambda_{\pi} \cap \Lambda_{l}$, то есть

$$
\log \chi_{\Lambda_{\pi}} \cap \Lambda_{l}(n) \gtrsim c_{\tau} n 2^{n-2} .
$$

Нижняя оценка $\tau\left(n \mid \mu_{\Lambda_{\pi}} \cap \Lambda_{L}=1\right)$ основана на построении специальной функции $n$ переменных по функции $f \in P_{n-3}$, имеющей максимальное число тупиковых комплексов.

Определим функцию $\Psi_{f} \in P_{n}$ следующим образом:

$$
\Psi_{f}\left(x_{1}, \ldots, x_{n}\right)=\Psi_{f, 0,0}\left(x_{1}, \ldots, x_{n}\right) \vee \Psi_{f, 0,1}\left(x_{1}, \ldots, x_{n}\right) \vee \Psi_{f, 1,1}\left(x_{1}, \ldots, x_{n}\right),
$$

где

$$
\begin{aligned}
\Psi_{f, 0,1}\left(x_{1}, \ldots, x_{n}\right)= & \bar{x}_{n-1} \cdot x_{n} \cdot\left(\bar{x}_{n-2} \vee x_{n-2} \cdot f\left(x_{1}, \ldots, x_{n-3}\right)\right), \\
\Psi_{f, 0,0}\left(x_{1}, \ldots, x_{n}\right)= & \bar{x}_{n-1} \cdot \bar{x}_{n} \cdot\left(x_{1} \oplus \ldots \oplus x_{n-2} \oplus 1\right) \cdot\left(\bar{x}_{n-2} \cdot \bar{f}\left(x_{1}, \ldots, x_{n-3}\right)\right. \\
& \left.\vee x_{n-2} \cdot f\left(x_{1}, \ldots, x_{n-3}\right)\right), \\
\Psi_{f, 1,1}\left(x_{1}, \ldots, x_{n}\right)= & x_{n-1} \cdot x_{n} \cdot\left(x_{1} \oplus \ldots \oplus x_{n-2}\right) \cdot\left(\bar{x}_{n-2} \cdot \bar{f}\left(x_{1}, \ldots, x_{n-3}\right)\right. \\
& \vee x_{n-2} \cdot f\left(x_{1}, \ldots, x_{n-3}\right) .
\end{aligned}
$$




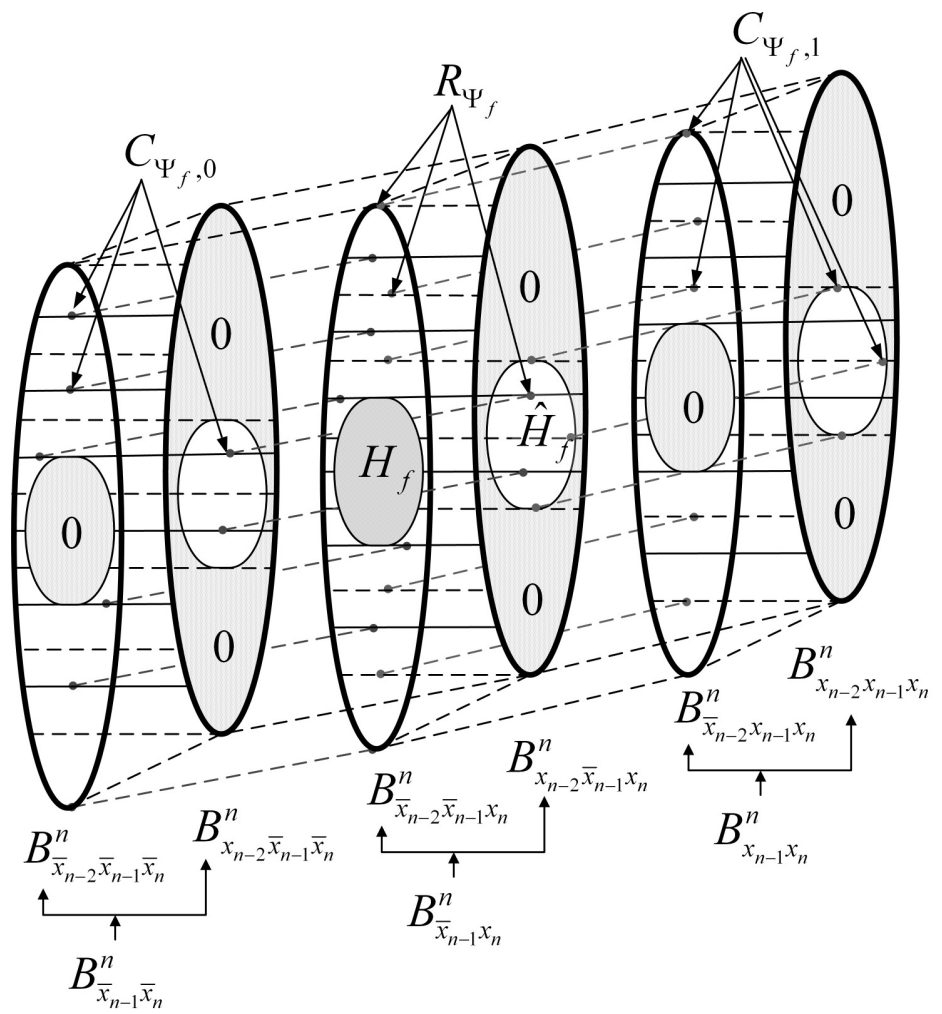

Рис. 2. Определение функции $\Psi_{f}$

Для функции $\Psi_{f}\left(x_{1}, \ldots, x_{n}\right)$ введем следующие подмножества вершин куба $B^{n}$ (см. рис. 2):

$$
\begin{aligned}
& H_{f}=\left\{\tilde{x} \in B^{n} \mid \bar{x}_{n-2} \cdot \bar{x}_{n-1} \cdot x_{n} \cdot f\left(x_{1}, \ldots, x_{n-3}\right)=1\right\} \subset B_{\bar{x}_{n-2} \bar{x}_{n-1} x_{n}}^{n}, \\
& \hat{H}_{f}=\left\{\tilde{x} \in B^{n} \mid x_{n-2} \cdot \bar{x}_{n-1} \cdot x_{n} \cdot f\left(x_{1}, \ldots, x_{n-3}\right)=1\right\} \subset B_{x_{n-2} \bar{x}_{n-1} x_{n}}^{n},
\end{aligned}
$$

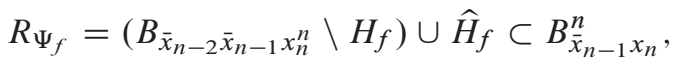

$$
\begin{aligned}
& C_{\Psi_{f}}=C_{\Psi_{f, 0}} \cup C_{\Psi_{f}, 1} \text {, }
\end{aligned}
$$

где

$$
\begin{aligned}
& C_{\Psi_{f}, 0}=\left\{\tilde{x} \in B^{n} \mid \Psi_{f, 0,0}\left(x_{1}, \ldots, x_{n}\right)=1\right\} \subset B_{\bar{x}_{n-1} \bar{x}_{n}}^{n}, \\
& C_{\Psi_{f}, 1}=\left\{\tilde{x} \in B^{n} \mid \Psi_{f, 1,1}\left(x_{1}, \ldots, x_{n}\right)=1\right\} \subset B_{x_{n-1} x_{n}}^{n} .
\end{aligned}
$$

Множество вершин $C_{\Psi_{f}}$ принадлежит либо четным слоям грани $B_{\bar{x}_{n-1}}^{n} \bar{x}_{n}$, либо нечетным слоям грани $B_{x_{n-1} x_{n}}^{n}$, так как является подмножеством вершин линейной функции. Для каждой вершины множества $C_{\Psi_{f}}$ существует единственная максимальная грань, содержащая эту вершину, которая является одномерной ядровой гранью функции $\Psi_{f}$. Сoответственно, каждая вершина из множества $C_{\Psi_{f}}$ является собственной вершиной одно- 
мерной ядровой грани, при этом вторая вершина в ядровой грани принадлежит множеству $R_{\Psi_{f}}$.

Обозначим через $\operatorname{Ker}\left(\Psi_{f}\right)$ комплекс граней, который состоит из всех ядровых одномерных граней функции $\Psi_{f}$ с собственными вершинами из множества $C_{\Psi_{f}}$. Вершины из множества $R_{\Psi_{f}}$ являются регулярными для ядровых граней комплекса $\operatorname{Ker}\left(\Psi_{f}\right)$.

Для любой меры сложности $\mathscr{L} \in \Lambda_{L}$ в любом $\mathscr{L}$-минимальном комплексе все грани являются максимальными. Максимальными гранями функции $\Psi_{f}$ являются: одномерные ядровые грани размерности 1 из комплекса $\operatorname{Ker}\left(\Psi_{f}\right)$, грань $B_{\bar{x}_{n-2}}^{n} \bar{x}_{n-1} x_{n}$ ранга 3 и любая грань $B_{i_{1}, \ldots, i_{k}, n-1, n}^{n, \sigma_{1}, \ldots, \sigma_{k}, 0,1} \subseteq B^{n}$, где $1 \leqslant i_{j} \leqslant n-3$ для $j=1, \ldots, k$, если грань $B_{i_{1}, \ldots, i_{k}}^{n-3, \sigma_{1}, \ldots, \sigma_{k}} \subseteq B^{n-3}$ является максимальной гранью функции $f$. Отметим, что для любой

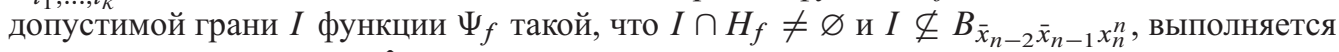
соотношение $I \subseteq H_{f} \cup \hat{H}_{f}$.

Лемма 5. Если $\tau(f)>1$ для функциии $f \in P_{n-3}$, где $n>5$, то

$$
\mu_{\mathscr{L}}\left(\Psi_{f}\right)=1, \quad \tau\left(\Psi_{f}\right)>\tau(f)
$$

для любой меры сложности $\mathscr{L} \in \Lambda_{\pi} \cap \Lambda_{L}$.

Для функции $f \in P_{n-3}$, имеющей максимальное число тупиковых комплексов,

$$
\tau(f)=\tau(n-3)>1
$$

при $n>5$. Тогда из леммы 5 следует, что

$$
\tau\left(\Psi_{f}\right)>\tau(n-3)
$$

И

$$
\mu_{\mathscr{L}}\left(\Psi_{f}\right)=1
$$

для любой меры сложности $\mathscr{L} \in \Lambda_{\pi} \cap \Lambda_{L}$, при этом единственный $\mathscr{L}$-минимальный комплекс является одинаковым для всех мер сложности. Это означает, что справедлива оценка

$$
\tau\left(n \mid \mu_{\Lambda_{\pi} \cap \Lambda_{L}}=1\right)>\tau(n-3)
$$

при $n>5$.

Нижние оценки числа функций из $P_{n}$ вида $2^{c 2^{n}}$, где $0<c<1$, для которых характеристики $\tau(f), \chi_{\Lambda_{\pi} \cap \Lambda_{l}}(f), \tau\left(f \mid \mu_{\Lambda_{\pi} \cap \Lambda_{L}}(f)=1\right)$ имеют порядок роста логарифма, равный $n 2^{n}$, основаны на работе [12] и используют некоторую конструкцию для построения тупиковых комплексов граней из множества $\mathscr{T}_{m-k, m}^{n} \cup \mathscr{T}_{n-m, n-m+k}^{n}$. Эта конструкция позволяет определять различные множества тупиковых комплексов для заданных множеств собственных вершин, которым соответствуют различные функции из множества

$$
\mathscr{F}_{m-k, m}^{n} \vee \mathscr{F}_{n-m, n-m+k}^{n}=\left\{f \mid f=f_{1} \vee f_{2}, f_{1} \in \mathscr{F}_{m-k, m}^{n}, f_{2} \in \mathscr{F}_{n-m, n-m+k}^{n}\right\} .
$$

Определим для

$$
m=\lfloor n / 2\rfloor-1, \quad k=\lfloor 0,1052 m\rfloor
$$

множество функций

$$
\mathscr{P}_{n, m, k}(t)=\left\{f \in \mathscr{F}_{m-k, m}^{n} \vee \mathscr{F}_{n-m, n-m+k}^{n} \mid \log \tau(f) \gtrsim t c_{\tau} n 2^{n}\right\} \subset P_{n} .
$$


Тогда

$$
\log \left|\mathscr{P}_{n, m, k}(t)\right| \asymp 2^{n}
$$

при $n \rightarrow \infty$ для $0<t<1$.

При преобразованиях $\Phi$ и $\Psi$ различные функции преобразуются в различные функции из $P_{n}$ в силу справедливости следующих утверждений.

Лемма 6. Если функциии $f, g \in P_{n-2} u f \neq g$, то $\Phi_{f} \neq \Phi_{g}$.

Лемма 7. Если функции $f, g \in P_{n-3} u f \neq g$, то $\Psi_{f} \neq \Psi_{g}$.

При применении к функциям множества $\mathscr{P}_{n-2, m, k}(t)$ преобразования $\Phi$ получим множество различных функций $n$ переменных, для которых

$$
\log \chi_{\Lambda_{\pi}} \cap \Lambda_{l}(f) \gtrsim t c_{\tau}, \quad n 2^{n-2}
$$

и логарифм числа таких функций имеет порядок роста $2^{n}$ при $n \rightarrow \infty$.

При применении к функциям множества $\mathscr{P}_{n-3, m, k}(t)$ преобразования $\Psi$ получим множество различных функций $n$ переменных, для которых

$$
\tau\left(f \mid \mu_{\Lambda_{\pi} \cap \Lambda_{L}}(f)=1\right) \gtrsim t c_{\tau} n 2^{n-3}
$$

и логарифм числа таких функций имеет порядок роста $2^{n}$ при $n \rightarrow \infty$.

\section{3. Формулировка основных результатов}

Используемые в оценках константы определим следующим образом:

$$
c_{\tau}=\frac{1}{2} C(x, y, \lambda), \quad \sigma_{\tau}=\varphi(\lambda, y),
$$

где

$$
\begin{aligned}
C(x, y, \lambda) & =y \varphi(\lambda, y) H(x / y), \\
\varphi(\lambda, y) & =\left(1-e^{-\lambda}\right)\left(1-\frac{\lambda y}{1-2 y}\right), \\
H(x) & =-x \log x-(1-x) \log (1-x) .
\end{aligned}
$$

При значениях параметров $x=0,1052, y=0,2104$ и $\lambda=1,0087$ находим, что

$$
c_{\tau}>1,355 \cdot 2^{-5}, \quad \sigma_{\tau}>0,4025 .
$$

Теорема 1. Для любой меры сложности $\mathscr{L} \in \Lambda_{\pi} \cap \Lambda_{l}$ при $n \rightarrow \infty$ справедливы оценки

$$
\frac{1}{4} c_{\tau} n 2^{n} \lesssim \log \chi_{\Lambda_{\pi} \cap \Lambda_{l}}(n) \leqslant \log \chi_{\mathscr{L}}(n)<\log \frac{3}{2} n 2^{n}+2^{n} \log e .
$$

Из теоремы 1 следует, что параметры $\log \chi_{\Lambda_{\pi}} \cap \Lambda_{l}(n)$ и $\log \chi_{\mathscr{L}}(n)$ для любой меры сложности $\mathscr{L} \in \Lambda_{\pi} \cap \Lambda_{l}$ имеют порядок роста $n 2^{n}$ при $n \rightarrow \infty$.

Теорема 2. При $n>5$ справедлива оценка

$$
\tau\left(n \mid \mu_{\Lambda_{\pi} \cap \Lambda_{L}}=1\right)>\tau(n-3) .
$$


Теорема 3. Для любой меры сложности $\mathscr{L} \in \Lambda_{\pi} \cap \Lambda_{L}$ при $n \rightarrow \infty$ справедливы оценки

$$
\left.\frac{1}{8} c_{\tau} n 2^{n} \lesssim \log \tau(n) \mid \mu_{\Lambda_{\pi} \cap \Lambda_{L}}=1\right) \leqslant \log \tau\left(n \mid \mu_{\mathscr{L}}=1\right)<\log \frac{3}{2} n 2^{n}+2^{n} \log e .
$$

Из теоремы 3 следует, что $\log \tau\left(n \mid \mu_{\Lambda_{\pi} \cap \Lambda_{L}}=1\right)$ и $\log \tau\left(n \mid \mu_{\mathscr{L}}=1\right)$ для любой меры сложности $\mathscr{L} \in \Lambda_{\pi} \cap \Lambda_{L}$ имеют порядок роста $n 2^{n}$ при $n \rightarrow \infty$.

Введем следующие множества функций $n$ переменных, для которых нижние оценки исследуемых параметров по порядку логарифма совпадают с максимальными значениями:

$$
\begin{aligned}
P_{n}(t, \tau) & =\left\{f \in P_{n} \mid \log \tau(f) \gtrsim t c_{\tau} n 2^{n}\right\}, \\
P_{n}\left(t, \chi_{\Lambda_{\pi}} \cap \Lambda_{l}\right) & =\left\{f \in P_{n} \mid \log \chi \Lambda_{\pi} \cap \Lambda_{l}(f) \gtrsim t c_{\tau} n 2^{n-2}\right\}, \\
P_{n}\left(t, \tau \mid \mu_{\Lambda_{\pi} \cap \Lambda_{L}}=1\right) & =\left\{f \in P_{n} \mid \log \tau(f) \gtrsim t c_{\tau} n 2^{n-3}, \mu_{\Lambda_{\pi}} \cap \Lambda_{L}(f)=1\right\},
\end{aligned}
$$

где $0<t<1$. Число функций в этих подмножествах обозначим соответственно через $p_{n}(t, \tau), p_{n}\left(t, \chi \Lambda_{\pi} \cap \Lambda_{l}\right)$ и $p_{n}\left(t, \tau \mid \mu_{\Lambda_{\pi}} \cap \Lambda_{L}=1\right)$.

Теорема 4. При $n \rightarrow \infty$ справедливы оценки

$$
\begin{aligned}
\log p_{n}(t, \tau) & \gtrsim \sigma_{\tau} \hbar(t) 2^{n}, \\
\log p_{n}\left(t, \chi_{\Lambda_{\pi} \cap \Lambda_{l}}\right) & \gtrsim \sigma_{\tau} \hbar(t) 2^{n-2}, \\
\log p_{n}\left(t, \tau \mid \mu_{\Lambda_{\pi} \cap \Lambda_{L}}=1\right) & \gtrsim \sigma_{\tau} \hbar(t) 2^{n-3},
\end{aligned}
$$

где

$$
\hbar(t)= \begin{cases}1, & 0<t \leqslant 1 / 2 \\ H(t), & 1 / 2<t<1\end{cases}
$$

Из теоремы 4 следует, что мощность множеств таких функций по порядку логарифма равна $2^{n}$, то есть совпадает с числом функций от $n$ переменных.

\section{4. Доказательства}

Доказательство леммы 1. Для любой функции, любого класса мер сложности С и любой меры сложности $\mathscr{L} \in \mathbf{C}$ выполняются неравенства

$$
\begin{gathered}
\chi_{\mathbf{C}}(f) \leqslant \chi_{\mathscr{L}}(f) \leqslant \tau(f), \\
\tau\left(f \mid \mu_{\mathbf{C}}=1\right) \leqslant \tau\left(f \mid \mu_{\mathscr{L}}=1\right) \leqslant \tau(f) .
\end{gathered}
$$

Поэтому

$$
\begin{gathered}
\chi_{\mathbf{C}}(n) \leqslant \chi_{\mathscr{L}}(n) \leqslant \tau(n), \\
\tau\left(n \mid \mu_{\mathbf{C}}=1\right) \leqslant \tau\left(n \mid \mu_{\mathscr{L}}=1\right) \leqslant \tau(n) .
\end{gathered}
$$

Обозначим через $\varphi^{n}(m)$ множество комплексов и через

$$
G_{n}(m)=\sum_{s=1}^{m}\left(\begin{array}{l}
3^{n} \\
s
\end{array}\right)
$$


число комплексов из не более, чем $m$ граней в единичном кубе $B^{n}$. Тупиковые комплексы являются неприводимыми, поэтому число граней в них не превосходит $2^{n}$, то есть

$$
\mathscr{T}(f) \subset \mathscr{T}^{n} \subseteq \mathscr{G}^{n}\left(2^{n}\right)
$$

для любой функции $f \in P_{n}$. Тогда

$$
\begin{aligned}
\log G_{n}(m) & <3^{n} H\left(m / 3^{n}\right), & m & <3^{n} / 2, \\
H(x) & <x \log (e / x), & 0 & <x<1 .
\end{aligned}
$$

Следовательно, при $2^{n}<3^{n} / 2$ или $n>1$ справедливы неравенства

$$
\log \tau(n)<\log T(n) \leqslant \log G_{n}\left(2^{n}\right)<n 2^{n} \log (3 / 2)+2^{n} \log e,
$$

и $\tau(1)=1$ при $n=1$, то есть оценка также справедлива. Лемма доказана.

Доказательство леммы 2. Произвольный комплекс $w$ функции $\Phi_{f}$ однозначно представляется в виде прямого объединения комплексов граней

$$
w=w\left(C_{\Phi_{f}}\right) \cup w^{\prime}\left(C_{\Phi_{f}}\right) \cup w\left(H_{f}\right) \cup w\left(R_{\Phi_{f}}\right),
$$

где

$w\left(C_{\Phi_{f}}\right)$ есть комплекс из $\left|C_{\Phi_{f}}\right|$ граней, которые содержат все вершины множества $C_{\Phi_{f}}$; в этот комплекс для каждой вершины из множества $C_{\Phi_{f}}$ входит либо 1-мерная ядровая грань из комплекса $\operatorname{Ker}\left(\Phi_{f}\right)$, либо 0-мерная грань, которая совпадает с вершиной;

$w^{\prime}\left(C_{\Phi_{f}}\right)$ есть комплекс граней, которые содержат вершины множества $C_{\Phi_{f}}$ и не входят в комплекс $w\left(C_{\Phi_{f}}\right)$;

$w\left(H_{f}\right)$ есть комплекс граней, которые содержат вершины из множества $H_{f}$; в этот комплекс могут входить только грани, допустимые для множества $S_{\Phi_{f}}$ и содержащие хотя бы одну вершину из множества $H_{f}$;

$w\left(R_{\Phi_{f}}\right)$ есть комплекс граней, которые не содержат вершин из множеств $C_{\Phi_{f}}$ и $H_{f}$; в этот комплекс могут входить только грани, допустимые для множества $R_{\Phi_{f}}$, то есть все вершины этих граней являются регулярными относительно ядровых граней комплекса $\operatorname{Ker}\left(\Phi_{f}\right)$.

В комплексе $w$ уменьшим ранг (увеличим размерность) граней из $w\left(C_{\Phi_{f}}\right) \cup w\left(H_{f}\right)$ до минимального ранга (максимальной размерности) для множества $N_{\Phi_{f}}$. При этом все грани из $w\left(C_{\Phi_{f}}\right)$ станут 1-мерными ядровыми гранями комплекса $\operatorname{Ker}\left(\Phi_{f}\right)$, а все грани из $w\left(H_{f}\right)$ станут $k$-мерными гранями, максимальными для множества $S_{\Phi_{f}}$. В силу аксиомы монотонности, $\mathscr{L}$-сложность полученного комплекса

$$
v=\operatorname{Ker}\left(\Phi_{f}\right) \cup w^{\prime}\left(C_{\Phi_{f}}\right) \cup v\left(H_{f}\right) \cup w\left(R_{\Phi_{f}}\right)
$$

не увеличится, то есть

$$
\mathscr{L}(w) \geqslant \mathscr{L}(v) .
$$

Очевидно, что если $w \notin \Omega^{n}\left(\Phi_{f}\right)$, то $v \notin \Omega^{n}\left(\Phi_{f}\right)$. Покажем, что тогда из $v$ можно получить удалением некоторых граней комплекс функции $\Phi_{f}$ меньшей $\mathscr{L}$-сложности для 
меры сложности $\mathscr{L} \in \Lambda_{\pi} \cap \Lambda_{l}$. Это будет означать, что комплексы $v$ и $w$ не могут быть $\mathscr{L}$-минимальными для функции $\Phi_{f}$.

Рассмотрим случай

$$
w^{\prime}\left(C_{\Phi_{f}}\right) \cup w\left(R_{\Phi_{f}}\right) \neq \varnothing
$$

Тогда $\operatorname{Ker}\left(\Phi_{f}\right) \cup v\left(H_{f}\right)$ является комплексом функции $\Phi_{f}$, так как все вершины из множества $C_{\Phi_{f}} \cup R_{\Phi_{f}}$ содержатся в гранях комплекса $\operatorname{Ker}\left(\Phi_{f}\right)$ и все вершины из множества $H_{f}$ содержатся в гранях комплекса $v\left(H_{f}\right)$. При удалении из комплекса $v$ всех граней, которые входят в $w^{\prime}\left(C_{\Phi_{f}}\right) \cup w\left(R_{\Phi_{f}}\right), \mathscr{L}$-сложность комплекса уменьшается, то есть

$$
\mathscr{L}(w) \geqslant \mathscr{L}(v)>\mathscr{L}\left(\operatorname{Ker}\left(\Phi_{f}\right) \cup v\left(H_{f}\right)\right)
$$

и комплекс $w$ не может быть $\mathscr{L}$-минимальным для функции $\Phi_{f}$.

Рассмотрим случай

$$
v=\operatorname{Ker}\left(\Phi_{f}\right) \cup v\left(H_{f}\right), \quad\left|v\left(H_{f}\right)\right|>\left(\begin{array}{c}
n-2 \\
m
\end{array}\right) .
$$

Рассмотрим комплекс граней $\operatorname{Ker}\left(\Phi_{f}\right) \cup M\left(S_{\Phi_{f}}\right)$ функции $\Phi_{f}$, где $M\left(S_{\Phi_{f}}\right)$ есть $L$-минимальный комплекс для $S_{\Phi_{f}}$, то есть для поясковой функции $S_{m-k, m}^{n-2}$ в грани $B_{\bar{x}_{n-1} x_{n}}^{n}$. Такой комплекс состоит (см. [10]) из $\left(\begin{array}{c}n-2 \\ m\end{array}\right)$ граней размерности $k, 0<k<m \leqslant\lfloor(n-2) / 2\rfloor$. Допустимо представление

$$
v\left(H_{f}\right)=v_{0}\left(H_{f}\right) \cup v^{\prime},
$$

где $v_{0}\left(H_{f}\right)-$ комплекс из некоторых $\left(\begin{array}{c}n-2 \\ m\end{array}\right)$ граней размерности $k$, максимальных для множества $S_{\Phi_{f}}$, а $v^{\prime}$ - непустой комплекс из оставшихся граней комплекса $v\left(H_{f}\right)$, которые не вошли в $v_{0}\left(H_{f}\right)$. Из комплекса $v_{0}\left(H_{f}\right)$ заменой граней на изоморфные может быть получен комплекс $M\left(S_{\Phi_{f}}\right)$, так как эти комплексы состоят из одинакового числа $k$-мерных граней, максимальных для пояса $S_{m-k, m}^{n-2}$ в $(n-2)$-мерной грани куба $B^{n}$. Поэтому для $\mathscr{L} \in \Lambda_{\pi}$

$$
\mathscr{L}\left(\operatorname{Ker}\left(\Phi_{f}\right) \cup v_{0}\left(H_{f}\right) \cup v^{\prime}\right)=\mathscr{L}\left(\operatorname{Ker}\left(\Phi_{f}\right) \cup M\left(S_{\Phi_{f}}\right) \cup v^{\prime}\right)
$$

и для $\mathscr{L} \in \Lambda_{l}$ при условии $v^{\prime} \neq \varnothing$

$$
\mathscr{L}\left(\operatorname{Ker}\left(\Phi_{f}\right) \cup M\left(S_{\Phi_{f}}\right) \cup v^{\prime}\right)>\mathscr{L}\left(\operatorname{Ker}\left(\Phi_{f}\right) \cup M\left(S_{\Phi_{f}}\right)\right) .
$$

Тогда

$$
\begin{aligned}
\mathscr{L}(w) \geqslant \mathscr{L}(v) & =\mathscr{L}\left(\operatorname{Ker}\left(\Phi_{f}\right) \cup v\left(H_{f}\right)\right) \\
& >\mathscr{L}\left(\operatorname{Ker}\left(\Phi_{f}\right) \cup M\left(S_{\Phi_{f}}\right),\right.
\end{aligned}
$$

и комплекс $w$ не может быть $\mathscr{L}$-минимальным для функции $\Phi_{f}$. Лемма доказана.

Доказательство леммы 3. Найдем нижнюю оценку числа тупиковых комплексов. Отображение

$$
\mathscr{I}\left(x_{1}, \ldots, x_{n-2}\right)=\left(x_{1}, \ldots, x_{n-2}, 0,1\right)
$$


определяет изоморфизм единичного куба $B^{n-2}$ и грани $B_{\bar{x}_{n-1} x_{n}}^{n}$ ранга 2 в кубе $B^{n}$, при котором сохраняется бинарное отношение смежности вершин в единичном кубе. Соответственно, сохраняются свойства принадлежности вершины подмножеству, максимальности грани для некоторого подмножества вершин, неприводимости комплекса граней, и другие.

При отображении $\mathscr{I}$ произвольная грань

$$
g=B_{i_{1}, \ldots, i_{r}}^{n-2, \sigma_{1}, \ldots, \sigma_{r}} \subset B^{n-2}
$$

отображается в грань

$$
\mathscr{I}(g)=B_{i_{1}, \ldots, i_{r}, n-1, n}^{n, \sigma_{1}, \ldots, \sigma_{r}, 0,1} \subset B_{\bar{x}_{n-1} x_{n}}^{n} \subset B^{n} .
$$

Для комплекса граней

$$
M=\left\{g_{j} \subset B^{n-2}, j=1, \ldots, l\right\}
$$

обозначим через

$$
\mathscr{I}(M)=\left\{\mathscr{I}\left(g_{j}\right), j=1, \ldots, l\right\}
$$

комплекс граней в грани $B_{\bar{x}_{n-1} x_{n}}^{n}$ куба $B^{n}$. При изоморфном отображении различные тупиковые комплексы граней в кубе $B^{n-2}$ преобразуются в различные тупиковые комплексы в кубе $B^{n}$.

Для функции $f \in \mathscr{F}_{m-k, m}^{n-2}$ множество тупиковых комплексов $\mathscr{T}(f) \subset \mathscr{T}_{m-k, m}^{n-2}$ и комплексы состоят из $k$-мерных максимальных граней пояса $S_{m-k, m}^{n-2} \subset B^{n-2}$. Тогда

$$
\mathscr{T}\left(H_{f}\right)=\{\mathscr{F}(T), T \in \mathscr{T}(f)\}
$$

- множество тупиковых комплексов $k$-мерных граней для подмножества $H_{f} \subseteq S_{\Phi_{f}} \subset B^{n}$. Bce $k$-мерные грани для подмножества вершин $H_{f}$ будут максимальными и для функции $\Phi_{f}$, так как множество $S_{\Phi_{f}}$ совпадает с поясом ширины $k$ в грани $B_{\bar{x}_{n-1} x_{n}}^{n}$. Поэтому любой комплекс из прямого объединения $\mathscr{T}\left(H_{f}\right) \cup \operatorname{Ker}\left(\Phi_{f}\right)$ является тупиковым для функции $\Phi_{f}$. Действительно, в таком комплексе ядровые грани из множества $\operatorname{Ker}\left(\Phi_{f}\right)$ содержат множество вершин $C_{\Phi_{f}} \cup R_{\Phi_{f}}$, и грани любого комплекса из $\mathcal{T}\left(H_{f}\right)$ содержат множество вершин $H_{f}$. При этом

$$
H_{f} \cap\left(C_{\Phi_{f}} \cup R_{\Phi_{f}}\right)=\varnothing,
$$

все грани являются максимальными и имеют собственные вершины. Поэтому

$$
\mathscr{T}\left(H_{f}\right) \cup \operatorname{Ker}\left(\Phi_{f}\right) \subseteq \mathscr{T}\left(\Phi_{f}\right)
$$

и, следовательно,

$$
\tau\left(\Phi_{f}\right) \geqslant \tau(f) .
$$

Найдем верхнюю оценку числа минимальных комплексов. В силу леммы 1,

$$
\mu_{\mathscr{L}}\left(\Phi_{f}\right) \subseteq \Omega^{n}\left(\Phi_{f}\right)=\varphi^{n}\left(C_{\Phi_{f}}\right) \cup \varphi^{n}\left(S_{\Phi_{f}}\right) .
$$


Поэтому

$$
\left|\mathcal{M}_{\mathscr{L}}\left(\Phi_{f}\right)\right| \leqslant\left|\Omega^{n}\left(\Phi_{f}\right)\right|=\left|\varphi^{n}\left(C_{\Phi_{f}}\right)\right|\left|\varphi^{n}\left(S_{\Phi_{f}}\right)\right| .
$$

Очевидно, что

$$
\left|\mathscr{G}^{n}\left(C_{\Phi_{f}}\right)\right| \leqslant 2^{\left|C_{\Phi_{f}}\right|}<2^{2^{n}}
$$

и

$$
\left|\varphi^{n}\left(S_{\Phi_{f}}\right)\right|<\sum_{i=1}^{b_{m}^{n-2}}\left(\begin{array}{c}
3^{n} \\
b_{m}^{n-2}
\end{array}\right)
$$

Так как

$$
b_{m}^{n-2}=\left(\begin{array}{c}
n-2 \\
\lfloor(n-2) / 2\rfloor-1
\end{array}\right)=O\left(2^{n} / \sqrt{n}\right)=o\left(3^{n}\right),
$$

получаем, что

$$
\begin{aligned}
\log \left|\varphi^{n}\left(S_{\Phi_{f}}\right)\right| & <3^{n} H\left(b_{m}^{n-2} / 3^{n}\right) \\
& <b_{m}^{n-2} \log \left(e 3^{n} / b_{m}^{n-2}\right)=O\left(\sqrt{n} 2^{n}\right),
\end{aligned}
$$

и, следовательно,

$$
\mu_{\mathscr{L}}\left(\Phi_{f}\right) \leqslant 2^{c \sqrt{n} 2^{n}}
$$

при $n \rightarrow \infty$, где $c-$ некоторая положительная постоянная. Лемма доказана.

Доказательство леммы 4. Пусть $w-$ произвольный комплекс граней функции $\Phi_{f} \vee \Phi_{f}^{*}$. Комплекс $w$ однозначно представляется в виде прямого объединения $w=w_{1} \cup w_{2}$, где $w_{1}$ и $w_{2}-$ комплексы граней для функций $\Phi_{f}$ и $\Phi_{f}^{*}$, которые являются несвязанными компонентами. Если $w \notin \Omega^{n}\left(\Phi_{f}\right) \cup \Omega^{n}\left(\Phi_{f}^{*}\right)$, то либо $w_{1} \notin \Omega^{n}\left(\Phi_{f}\right)$, либо $w_{2} \notin \Omega^{n}\left(\Phi_{f}^{*}\right)$. Отметим, что при удалении грани из любого комплекса граней функции $\Phi_{f} \vee \Phi_{f}^{*}$ сложность комплекса строго уменьшается для любой меры сложности из $\Lambda_{l}$.

В случае $w_{1} \notin \Omega^{n}\left(\Phi_{f}\right)$ покажем, что из $w_{1}$ может быть получен такой комплекс $\widehat{w}_{1}$, что комплекс $\widehat{w}_{1} \cup w_{2}$ функции $\Phi_{f} \vee \Phi_{f}^{*}$ будет иметь меньшую $\mathscr{L}$-сложность, чем комплекс $w=w_{1} \cup w_{2}$. Это будет означать, что комплекс $w$ не может быть $\mathscr{L}$-минимальным для функции $\Phi_{f} \vee \Phi_{f}^{*}$.

Аналогично доказательству леммы 1 , комплекс $w_{1}$ функции $\Phi_{f}$ однозначно представляется в виде прямого объединения комплексов граней

$$
w_{1}=w_{1}\left(C_{\Phi_{f}}\right) \cup w_{1}^{\prime}\left(C_{\Phi_{f}}\right) \cup w_{1}\left(H_{f}\right) \cup w_{1}\left(R_{\Phi_{f}}\right) .
$$

В комплексе $w_{1}$ увеличим размерность граней комплекса $w_{1}\left(C_{\Phi_{f}}\right) \cup w_{1}\left(H_{f}\right)$ до максимальной размерности. В силу аксиомы монотонности, $\mathscr{L}$-сложность полученного комплекса $v_{1} \cup w_{2}$, где

$$
v_{1}=\operatorname{Ker}\left(\Phi_{f}\right) \cup w_{1}^{\prime}\left(C_{\Phi_{f}}\right) \cup v_{1}\left(H_{f}\right) \cup w_{1}\left(R_{\Phi_{f}}\right),
$$

не увеличится, то есть

$$
\mathscr{L}(w) \geqslant \mathscr{L}\left(v_{1} \cup w_{2}\right) .
$$

3 Дискретная математика, т.24 №2 
Рассмотрим теперь случай

$$
w_{1}^{\prime}\left(C_{\Phi_{f}}\right) \cup w_{1}\left(R_{\Phi_{f}}\right) \neq \varnothing .
$$

Тогда $\operatorname{Ker}\left(\Phi_{f}\right) \cup v_{1}\left(H_{f}\right)$ является комплексом функции $\Phi_{f}$, и $\mathscr{L}$-сложность комплекса $v_{1}$ уменьшается при удалении граней комплекса $w^{\prime}\left(C_{\Phi_{f}}\right) \cup w\left(R_{\Phi_{f}}\right)$. Поэтому

$$
\mathscr{L}(w) \geqslant \mathscr{L}\left(v_{1} \cup w_{2}\right)>\mathscr{L}\left(\operatorname{Ker}\left(\Phi_{f}\right) \cup v\left(H_{f}\right) \cup w_{2}\right),
$$

и комплекс $w$ не может быть $\mathscr{L}$-минимальным для функции $\Phi_{f} \vee \Phi_{f}^{*}$.

Рассмотрим случай

$$
v_{1}=\operatorname{Ker}\left(\Phi_{f}\right) \cup v_{1}\left(H_{f}\right), \quad\left|v_{1}\left(H_{f}\right)\right|>\left(\begin{array}{c}
n-2 \\
m
\end{array}\right) .
$$

Допустимо представление

$$
v_{1}\left(H_{f}\right)=\hat{v}_{1}\left(H_{f}\right) \cup v_{1}^{\prime},
$$

где $\hat{v}_{1}\left(H_{f}\right)-$ комплекс из $\left(\begin{array}{c}n-2 \\ m\end{array}\right)$ граней размерности $k$, максимальных для множества $S_{\Phi_{f}}$, и $v_{1}^{\prime}-$ непустой комплекс из граней комплекса $v_{1}\left(H_{f}\right)$, не вошедших в $\hat{v}_{1}\left(H_{f}\right)$. Из комплекса $\hat{v}_{1}\left(H_{f}\right)$ заменой граней на изоморфные может быть получен комплекс $M\left(S_{\Phi_{f}}\right)$ - $L$-минимальный комплекс для множества $S_{\Phi_{f}}$. Поэтому для $\mathscr{L} \in \Lambda_{\pi}$

$$
\mathscr{L}\left(\operatorname{Ker}\left(\Phi_{f}\right) \cup \hat{v}_{1}\left(H_{f}\right) \cup v_{1}^{\prime} \cup w_{2}\right)=\mathscr{L}\left(\operatorname{Ker}\left(\Phi_{f}\right) \cup M\left(S_{\Phi_{f}}\right) \cup v_{1}^{\prime} \cup w_{2}\right),
$$

и для $\mathscr{L} \in \Lambda_{l}$ при условии $v_{1}^{\prime} \neq \varnothing$

$$
\mathscr{L}\left(\operatorname{Ker}\left(\Phi_{f}\right) \cup M\left(S_{\Phi_{f}}\right) \cup v_{1}^{\prime} \cup w_{2}\right)>\mathscr{L}\left(\operatorname{Ker}\left(\Phi_{f}\right) \cup M\left(S_{\Phi_{f}}\right) \cup w_{2}\right) .
$$

Тогда

$$
\begin{aligned}
\mathscr{L}(w) & \geqslant \mathscr{L}\left(v_{1} \cup w_{2}\right) \\
& =\mathscr{L}\left(\operatorname{Ker}\left(\Phi_{f}\right) \cup v_{1}\left(H_{f}\right) \cup w_{2}\right)>\mathscr{L}\left(\operatorname{Ker}\left(\Phi_{f}\right) \cup M\left(S_{\Phi_{f}}\right) \cup w_{2}\right),
\end{aligned}
$$

и комплекс $w$ не может быть $\mathscr{L}$-минимальным для функции $\Phi_{f} \vee \Phi_{f}^{*}$.

В случае $w_{2} \notin \Omega^{n}\left(\Phi_{f}^{*}\right)$ доказательство выполняется аналогично: уменьшением ранга и удалением некоторых граней из $w_{2}$ может быть получен такой комплекс $\widehat{w}_{2}$, что комплекс $w_{1} \cup \widehat{w}_{2}$ функции $\Phi_{f} \vee \Phi_{f}^{*}$ будет иметь меньшую $\mathscr{L}$-сложность, чем комплекс $w=w_{1} \cup w_{2}$. Поэтому комплекс $w$ не может быть $\mathscr{L}$-минимальным для функции $\Phi_{f} \vee \Phi_{f}^{*}$. Лемма доказана.

Доказательство теоремы 1. Верхние оценки являются следствием леммы 1. Нижняя оценка вытекает из существования функции $f \in \underset{m-k, m}{\mathscr{F}_{m}-2}$, где $m=\lfloor(n-2) / 2\rfloor-1$ и $k=\lfloor 0,1052 m\rfloor$, для которой

$$
\log \tau(f) \gtrsim c_{\tau} n 2^{n-3} .
$$

Поэтому, в силу лемм 2-4, при $n \rightarrow \infty$ справедливы оценки

$$
\begin{aligned}
\log \tau\left(\Phi_{f} \vee \Phi_{f}^{*}\right) & \geqslant 2 \log \tau(f) \gtrsim 2 c_{\tau} n 2^{n-3}=c_{\tau} n 2^{n-2}, \\
\log \mu_{\mathscr{L}}\left(\Phi_{f} \vee \Phi_{f}^{*}\right) & =O\left(\sqrt{n} 2^{n}\right)
\end{aligned}
$$


для любой меры сложности $\mathscr{L} \in \Lambda_{\pi} \cap \Lambda_{l}$. Это означает, что для класса мер сложности $\Lambda_{\pi} \cap \Lambda_{l}$ справедлива нижняя оценка

$$
\log \chi_{\Lambda_{\pi} \cap \Lambda_{l}(n)} \gtrsim \frac{c_{\tau}}{4} n 2^{n}-C \sqrt{n} 2^{n} \sim \frac{c_{\tau}}{4} n 2^{n},
$$

здесь $C$ - некоторая положительная постоянная. Теорема доказана.

Доказательство леммы 5. Для меры сложности $\mathscr{L} \in \Lambda_{L}$ любой $\mathscr{L}$-минимальный комплекс является тупиковым, то есть все грани в нем максимальные. Поэтому в тупиковых и $\mathscr{L}$-минимальных комплексах функции $\Psi_{f}$ вершины множества $C_{\Psi_{f}}$ покрываются комплексом $\operatorname{Ker}\left(\Psi_{f}\right)$ - одномерными ядровыми гранями функции $\Psi_{f}$, для которых вершины множества $C_{\Psi_{f}}$ являются собственными. При этом

$$
N_{\Psi_{f}} \cap B_{\bar{x}_{n-1} x_{n}}^{n}=H_{f} \cup R_{\Psi_{f}}
$$

и

$$
H_{f} \cap R_{\Psi_{f}}=\varnothing
$$

где $R_{\Psi_{f}}$ есть множество регулярных вершин ядровых граней.

Для функции $f \in P_{n-3}$ из условия $\tau(f)>1$ следует, что множество $N_{f}$ не является гранью и монотонным множеством в $B^{n-3}$, так как в таких случаях $\tau(f)=1$. Следовательно, множество вершин $H_{f}$ в грани $B_{\bar{x}_{n-2} \bar{x}_{n-1} x_{n}}^{n}$ также не является гранью и монотонным множеством.

Сначала покажем, что

$$
\mathcal{M}_{\mathscr{L}}\left(\Psi_{f}\right)=\left\{B_{\bar{x}_{n-2} \bar{x}_{n-1} x_{n}}^{n} \cup \operatorname{Ker}\left(\Psi_{f}\right),\right.
$$

то есть

$$
\mu_{\mathscr{L}}\left(\Psi_{f}\right)=1
$$

Множество вершин $H_{f}$ в любом $\mathscr{L}$-минимальном комплексе содержится в гранях, которые не входят в множество $\operatorname{Ker}\left(\Psi_{f}\right)$.

Если

$$
w=\{I\} \cup \operatorname{Ker}\left(\Psi_{f}\right)
$$

есть тупиковый комплекс функции $\Psi_{f}$, где максимальная грань $I \neq B_{\bar{x}_{n-2}} \bar{x}_{n-1} x_{n}^{n}$, то

$$
I=H_{f} \cup \hat{H}_{f} .
$$

Тогда множество

$$
H_{f}=I \cap B_{\bar{x}_{n-2} \bar{x}_{n-1} x_{n}}^{n},
$$

является гранью, что невозможно при $\tau(f)>1$.

Если

$$
w=\left\{I_{1}, \ldots, I_{l}\right\} \cup \operatorname{Ker}\left(\Psi_{f}\right)
$$

есть тупиковый комплекс функции $\Psi_{f}$, где максимальные грани $I_{j} \neq B_{\bar{x}_{n-2}}^{n} \bar{x}_{n-1} x_{n}$ для $j=1, \ldots, l$ и $l>1$, то найдется грань $I \in\left\{I_{1}, \ldots, I_{l}\right\}$, для которой $I \subseteq B_{\bar{x}_{s}}^{n} \bar{x}_{n-1} x_{n}$ 
и $1 \leqslant s \leqslant n-3$. Действительно, любая максимальная грань $I$ функции $\Psi_{f}$, отличная от грани $B_{\bar{x}_{n-2}}^{n} \bar{x}_{n-1} x_{n}$ и содержащая вершину из множества $H_{f}$, содержится в множестве $H_{f} \cup \hat{H}_{f} \subset B_{\bar{x}_{n-1} x_{n}}^{n}$. Следовательно, $I \subseteq B_{x_{s}^{\sigma} \bar{x}_{n-1} x_{n}}^{n}$, где $1 \leqslant s \leqslant n-3$ и $\sigma \in\{0,1\}$. Так как множество вершин $H_{f}$ не является монотонным в грани $B_{\bar{x}_{n-2}}^{n} \bar{x}_{n-1} x_{n}$, то всегда существует

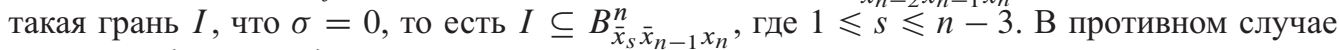
все грани $\left\{I_{1}, \ldots, I_{l}\right\}$ содержат максимальную вершину грани $B_{\bar{x}_{n-2}}^{n} \bar{x}_{n-1} x_{n}$ и множество вершин $H_{f}$ является монотонным в грани $B_{\bar{x}_{n-2} \bar{x}_{n-1} x_{n}}$.

Так как $\mathscr{L} \in \Lambda_{L}$, в силу аксиомы монотонности

$$
\begin{aligned}
\mathscr{L}(w) & =\mathscr{L}\left(\left\{I_{1}, I_{2}, \ldots\right\} \cup \operatorname{Ker}\left(\Psi_{f}\right)\right) \\
& >\mathscr{L}\left(\{I\} \cup \operatorname{Ker}\left(\Psi_{f}\right)\right) \geqslant \mathscr{L}\left(\left\{B_{\bar{x}_{s} \bar{x}_{n-1} x_{n}}^{n}\right\} \cup \operatorname{Ker}\left(\Psi_{f}\right)\right) .
\end{aligned}
$$

Так как $\mathscr{L} \in \Lambda_{\pi}$, верно, что

$$
\mathscr{L}\left(\left\{B_{\bar{x}_{s} \bar{x}_{n-1} x_{n}}^{n}\right\} \cup \operatorname{Ker}\left(\Psi_{f}\right)\right)=\mathscr{L}\left(\left\{B_{\bar{x}_{n-2} \bar{x}_{n-1} x_{n}}^{n}\right\} \cup \operatorname{Ker}\left(\Psi_{f}\right)\right) .
$$

Следовательно,

$$
\mathscr{L}(w)>\mathscr{L}\left(\left\{B_{\bar{x}_{n-2} \bar{x}_{n-1} x_{n}}^{n}\right\} \cup \operatorname{Ker}\left(\Psi_{f}\right)\right),
$$

и комплекс $w$ не является $\mathscr{L}$-минимальным для функции $\Psi_{f}$.

Теперь покажем, что

$$
\mathscr{T}\left(\Psi_{f}\right) \supset \mathscr{T}\left(H_{f} \cup \hat{H}_{f}\right) \cup \operatorname{Ker}\left(\Psi_{f}\right)
$$

И

$$
\tau\left(H_{f} \cup \hat{H}_{f}\right) \geqslant \tau(f) .
$$

Отображение

$$
\mathscr{I}\left(x_{1}, \ldots, x_{n-3}\right)=\left(x_{1}, \ldots, x_{n-3}, 0,0,1\right)
$$

определяет изоморфизм единичного куба $B^{n-3}$ и грани $B_{\bar{x}_{n-2}}^{n} \bar{x}_{n-1} x_{n} \subset B^{n}$ ранга 3 , при котором сохраняется бинарное отношение смежности вершин в единичном кубе. Соответственно, сохраняются свойства принадлежности вершины подмножеству, максимальности грани для некоторого подмножества вершин, неприводимости комплекса граней и другие.

При отображении $\mathscr{I}$ произвольная грань

$$
g=B_{i_{1}, \ldots, i_{r}}^{n-3, \sigma_{1}, \ldots, \sigma_{r}} \subset B^{n-3}
$$

отображается в грань

$$
\mathscr{I}(g)=B_{i_{1}, \ldots, i_{r}, n-2, n-1, n}^{n, \sigma_{1}, \ldots, \sigma_{r}, 0,0,1} \subseteq B_{\bar{x}_{n-2} \bar{x}_{n-1} x_{n}}^{n} \subset B^{n} .
$$

Для комплекса граней

$$
M=\left\{g_{j} \subset B^{n-3}, j=1, \ldots, l\right\}
$$

обозначим через

$$
\mathscr{I}(M)=\left\{\mathscr{I}\left(g_{j}\right), j=1, \ldots, l\right\}
$$


комплекс граней куба $B^{n}$, которые содержатся в грани $B_{\bar{x}_{n-2}}^{n} \bar{x}_{n-1} x_{n}$.

При изоморфном отображении различные тупиковые комплексы в кубе $B^{n-3}$ преобразуются в различные тупиковые комплексы в кубе $B^{n}$. Следовательно,

$$
\mathscr{T}\left(H_{f}\right)=\{\mathscr{T}(T), T \in \mathscr{T}(f)\}
$$

есть множество тупиковых комплексов для множества $H_{f} \subset B^{n}$.

Для грани $B_{i_{1}, \ldots, i_{r}, n-2, n-1, n}^{n, \sigma_{1}, \ldots, \sigma_{r}, 0,0,1} \subset B_{\bar{x}_{n-2} \bar{x}_{n-1} x_{n}}^{n}$ определим преобразование

$$
\mathscr{D}_{n-2}\left(B_{i_{1}, \ldots, i_{r}, n-2, n-1, n}^{n, \sigma_{1}, \ldots, \sigma_{r}, 0,0,1}\right)=B_{i_{1}, \ldots, i_{r}, n-1, n}^{n, \sigma_{1}, \ldots, \sigma_{r}, 0,1} \subset B_{\bar{x}_{n-1} x_{n}}^{n},
$$

получаемое при удалении координаты $n-2$ из направления грани.

Для комплекса граней

$$
M=\left\{g_{j} \subset B_{\bar{x}_{n-2} \bar{x}_{n-1} x_{n}}^{n}, j=1, \ldots, l\right\}
$$

обозначим через

$$
\mathscr{D}_{n-2}(M)=\left\{\mathscr{D}_{n-2}\left(g_{j}\right), j=1, \ldots, l\right\}
$$

комплекс граней куба $B^{n}$, содержащихся в грани $B_{\bar{x}_{n-1} x_{n}}^{n}$. Тогда $\left\{\mathscr{D}_{n-2}(T), T \in \mathscr{T}\left(H_{f}\right)\right\}$ - множество различных тупиковых комплексов для множества $H_{f} \cup \hat{H}_{f} \subseteq B_{\bar{x}_{n-1} x_{n}}^{n}$, так как каждая грань $\mathscr{D}_{n-2}(g)$ имеет собственную вершину, которая совпадает с собственной вершиной грани $g \in T$ и содержится в $H_{f} \subseteq B_{\bar{x}_{n-2} \bar{x}_{n-1} x_{n}}^{n}$. Следовательно,

$$
\mathscr{T}\left(H_{f} \cup \hat{H}_{f}\right) \supseteq\left\{\mathscr{D}_{n-2}(T), T \in \mathscr{T}\left(H_{f}\right)\right\}
$$

и

$$
\tau\left(H_{f} \cup \hat{H}_{f}\right) \geqslant \tau(f) .
$$

При объединении любого тупикового комплекса $T \in \mathscr{T}\left(H_{f} \cup \hat{H}_{f}\right)$ с комплексом ядровых граней $\operatorname{Ker}\left(\Psi_{f}\right)$ грани комплекса $T$ остаются максимальными, и их собственные вершины остаются собственными в объединенном комплексе, так как вершины из множества $B_{x_{n-2}}^{n} \bar{x}_{n-1} x_{n} \backslash \hat{H}_{f}$ и все вершины, соседние с вершинами из множества $H_{f}$ в гранях $B_{\bar{x}_{n-2}}^{n} \bar{x}_{n-1} x_{n}$ и $B_{\bar{x}_{n-2} x_{n-1} x_{n}}^{n}$, не принадлежат $N_{\Psi_{f}}$. Поэтому

$$
\begin{aligned}
& \mathscr{T}\left(\Psi_{f}\right) \supset M_{\mathscr{L}}\left(\Psi_{f}\right)=\left\{B_{\bar{x}_{n-2} \bar{x}_{n-1} x_{n}}^{n}\right\} \cup \operatorname{Ker}\left(\Psi_{f}\right), \\
& \mathscr{T}\left(\Psi_{f}\right) \supset \mathscr{T}\left(H_{f} \cup \hat{H}_{f}\right) \cup \operatorname{Ker}\left(\Psi_{f}\right), \quad M_{\mathscr{L}}\left(\Psi_{f}\right) \cap\left(\mathscr{T}\left(H_{f} \cup \hat{H}_{f}\right) \cup \operatorname{Ker}\left(\Psi_{f}\right)\right)=\varnothing .
\end{aligned}
$$

Тогда

$$
\tau\left(\Psi_{f}\right) \geqslant \tau\left(H_{f} \cup \hat{H}_{f}\right)+1>\tau(f) .
$$

Лемма доказана.

Доказательство теоремы 2. Пусть $f \in P_{n-3}$ и $\tau(f)=\tau(n-3)>1$ при $n>5$. Тогда из леммы 5 следует, что $\tau\left(\Psi_{f}\right)>\tau(f)=\tau(n-3)$ и $\mu_{\mathscr{L}}\left(\Psi_{f}\right)=1$ для любой меры сложности $\mathscr{L} \in \Lambda_{\pi} \cap \Lambda_{L}$. Следовательно,

$$
\tau\left(n \mid \mu_{\Lambda_{\pi} \cap \Lambda_{L}}=1\right) \geqslant \tau\left(\Psi_{f}\right)>\tau(n-3) .
$$

Теорема доказана. 
Доказательство теоремы 3. Верхние оценки являются следствием леммы 1. Нижняя оценка вытекает из теоремы 2 и оценки

$$
\log \tau(n-3) \gtrsim c_{\tau}(n-3) 2^{n-3} \sim c_{\tau} n 2^{n-3},
$$

из которой следует оценка

$$
\log \tau\left(n \mid \mu_{\Lambda_{\pi} \cap \Lambda_{L}}=1\right)>\log \tau(n-3) \gtrsim \frac{c_{\tau}}{8} n 2^{n} .
$$

Теорема доказана.

Доказательство леммы 6. Для функции $\Phi_{f} \in P_{n}$, где функция $f \in P_{n-2}$, определим подмножество вершин

$$
\Delta_{f}=\left\{\tilde{x} \in B^{n} \mid x_{n-1} \cdot x_{n} \cdot f\left(x_{1}, \ldots, x_{n-2}\right)=1\right\} \subset B^{n} \backslash N_{\Phi_{f}} .
$$

Для различных функций $f, g \in P_{n-2}$ верно, что $\Delta_{f} \neq \Delta_{g}$. Тогда $B^{n} \backslash N_{\Phi_{f}} \neq B^{n} \backslash N_{\Phi_{g}}$, то есть функции $\Phi_{f}$ и $\Phi_{g}$ являются различными. Лемма доказана.

Доказательство леммы 7. Для функции $\Psi_{f} \in P_{n}$, где функция $f \in P_{n-3}$, определим подмножество вершин

$$
\begin{aligned}
\Delta_{f} & =\hat{H}_{f}=N_{\Psi_{f}} \cap B_{x_{n-2} \bar{x}_{n-1} x_{n}} \\
& =\left\{\tilde{x} \in B^{n} \mid x_{n-2} \cdot \bar{x}_{n-1} \cdot x_{n} \cdot f\left(x_{1}, \ldots, x_{n-3}\right)=1\right\} .
\end{aligned}
$$

Для различных функций $f, g \in P_{n-3}$ верно, что $\Delta_{f} \neq \Delta_{g}$. Тогда $N_{\Psi_{f}} \neq N_{\Psi_{g}}$, то есть функции $\Psi_{f}$ и $\Psi_{g}$ являются различными. Лемма доказана.

Доказательство теоремы 4 . Получим нижнюю оценку $p_{n}(t, \tau)$. Определим подмножество функций $\mathscr{P}_{n, m, k}(t) \subseteq P_{n}(t, \tau) \subset P_{n}$, где

$$
\mathscr{P}_{n, m, k}(t)=\left\{f \in \mathscr{F}_{m-k, m}^{n} \vee \mathscr{F}_{n-m, n-m+k}^{n} \in P_{n} \mid \log \tau(f) \gtrsim t c_{\tau} n 2^{n}\right\} .
$$

Покажем, что

$$
\log p_{n}(t, \tau) \geqslant \log \left|\mathscr{P}_{n, m, k}(t)\right| \gtrsim \sigma_{\tau} \hbar(t) 2^{n}
$$

при $n \rightarrow \infty$ для $m=\lfloor n / 2\rfloor-1$ и $k=\lfloor 0,1052 m\rfloor$.

Для доказательства используется конструкция построения множеств тупиковых комплексов в поясе единичного куба, изложенная в [12]. Положим

$$
m=\lfloor n / 2\rfloor-1, \quad p=\lfloor\sqrt{n} \log n\rfloor, \quad k=\lfloor x m\rfloor, \quad k_{0}=\lfloor y m\rfloor,
$$

где

$$
x=0,1052, \quad y=0,2104, \quad \lambda=1,0087 .
$$

При указанных значениях параметров существует тупиковый комплекс $k_{0}$-мерных граней $T_{0} \in \mathscr{T}_{m-k_{0}, m}^{n}$ такой, что множество собственных вершин $C_{T_{0}} \subset S_{m-p, m}^{n}$ и

$$
\left|T_{0}\right|=\left|C_{T_{0}}\right| \gtrsim\left|S_{m-p, m}^{n}\right| \varphi(\lambda, y) \sim 2^{n-1} \varphi(\lambda, y) .
$$


Произвольное подмножество граней $T \subseteq T_{0}$ также образует тупиковый комплекс $T \in \mathscr{T}_{m-k_{0}, m}^{n}$ с множеством собственных вершин $C_{T} \subseteq C_{T_{0}} \subset S_{m-p, m}^{n}$. По тупиковому комплексу $T$ может быть определено множество тупиковых комплексов $k$-мерных граней $\Omega_{\tau}^{n}\left(T, C_{T}, S_{m-k_{0}, m}^{n}, p, k\right) \subseteq \mathscr{T}_{m-k, m}^{n}$. При этом, множеством собственных вершин граней в любом тупиковом комплексе является множество вершин $C_{T}$, и справедлива оценка

$$
\begin{aligned}
\log \left|\mathscr{T}_{m-k, m}^{n}\right| & \geqslant \log \left|\Omega_{\tau}^{n}\left(T, C_{T}, S_{m-k_{0}, m}^{n}, p, k\right)\right| \\
& \gtrsim|T|(n y / 2) H(x / y) \\
& \gtrsim\left(|T| /\left|T_{0}\right|\right) n 2^{n-2} C(x, y, \lambda) \\
& =\left(|T| /\left|T_{0}\right|\right) c_{\tau} n 2^{n-1},
\end{aligned}
$$

где

$$
C(x, y, \lambda)=y \varphi(\lambda, y) H(x / y)=2 c_{\tau} .
$$

Будем рассматривать тупиковые комплексы одновременно для двух подмножеств: $T_{0} \in \mathscr{T}_{m-k_{0}, m}^{n}$ в поясе $S_{m-k_{0}, m}^{n}$ ниже слоя $\lfloor n / 2\rfloor$, и $\mathscr{A}\left(T_{0}\right) \in \mathscr{T}_{n-m, n-m+k_{0}}^{n}$ в поясе $S_{n-m, n-m+k_{0}}^{n}$, расположенном выше слоя $\lfloor n / 2\rfloor$, где автоморфизм $\mathscr{A}: P_{n} \rightarrow P_{n}$ определяется отображением

$$
f_{\mathscr{A}}\left(x_{1}, \ldots, x_{n}\right)=f\left(\bar{x}_{1}, \ldots, \bar{x}_{n}\right) .
$$

Обозначим через $R\left(t, T_{0}\right)$ множество тупиковых комплексов, которые могут быть получены из тупикового комплекса $T_{0} \cup \mathscr{A}\left(T_{0}\right)$ выбором не менее, чем $t\left|T_{0} \cup \mathscr{A}\left(T_{0}\right)\right|$ граней. Произвольно выбранное подмножество граней $T \subseteq T_{0} \cup \mathcal{A}\left(T_{0}\right)$ образует тупиковый комплекс $T \in \mathscr{T}_{m-k_{0}, m}^{n} \cup \mathscr{T}_{n-m, n-m+k_{0}}^{n}$ с множеством собственных вершин $C_{T}$, при этом $C_{T} \cap C_{T_{0}} \subset S_{m-p, m}^{n}$ и $C_{T} \cap \mathscr{A}\left(C_{T_{0}}\right) \subset S_{n-m, n-m+p}^{n}$.

По тупиковому комплексу $T \in R\left(t, T_{0}\right)$ может быть построено множество тупиковых комплексов $k$-мерных граней $\Omega_{\tau}^{n}(T) \subseteq \mathscr{T}_{m-k, m}^{n} \cup \mathscr{T}_{n-m, n-m+k}^{n}$, которое представляется в виде прямого объединения двух множеств тупиковых комплексов для соответствующих подмножеств куба $B^{n}$ :

$$
\Omega_{\tau}^{n}\left(T \cap T_{0}, C_{T} \cap C_{T_{0}}, S_{m-k_{0}, m}^{n}, p, k\right) \subseteq \mathscr{T}_{m-k, m}^{n}
$$

и

$$
\Omega_{\tau}^{n}\left(T \cap \mathscr{A}\left(T_{0}\right), C_{T \cap C_{\mathscr{A}}\left(T_{0}\right)}, S_{n-m, n-m+k_{0}}^{n}, p, k\right) \subseteq \mathscr{T}_{n-m, n-m+k}^{n} .
$$

Так как

$$
\begin{aligned}
\left|C_{T} \cap C_{T_{0}}\right|+\left|C_{T} \cap C_{\mathscr{A}\left(T_{0}\right)}\right| & =\left|C_{T}\right| \\
& \geqslant t\left|C_{T_{0}} \cup C_{\mathscr{A}\left(T_{0}\right)}\right| \\
& =2 t\left|T_{0}\right|,
\end{aligned}
$$

при выбранных значениях параметров

$$
\begin{aligned}
\log \left|\Omega_{\tau}^{n}(T)\right| & \gtrsim\left(\left|C_{T} \cap C_{T_{0}}\right|+\left|C_{T} \cap C_{\mathscr{A}\left(T_{0}\right)}\right|\right)(n y / 2) H(x / y) \\
& \geqslant 2 t\left|T_{0}\right|(n y / 2) H(x / y) \\
& \gtrsim 2 t n 2^{n-2} C(x, y, \lambda) \\
& =t c_{\tau} n 2^{n} .
\end{aligned}
$$


Все комплексы из множества $\Omega_{\tau}^{n}(T)$ являются тупиковыми комплексами $k$-мерных граней из множества комплексов $\mathscr{T}_{m-k, m}^{n} \cup \mathscr{T}_{n-m, n-m+k}^{n}$. Поэтому существует такая функция $f_{T} \in \mathscr{F}_{m-k, m}^{n} \vee \mathscr{F}_{n-m, n-m+k}^{n}$, чTO

$$
\begin{aligned}
\log \tau\left(f_{T}\right) & \geqslant \log \left(2^{-2^{n}}\left|\Omega_{\tau}^{n}(T)\right|\right) \\
& \gtrsim t c_{\tau} n 2^{n}-2^{n} \sim t c_{\tau} n 2^{n} .
\end{aligned}
$$

Тупиковые комплексы для различных подмножеств $T \in R\left(t, T_{0}\right)$ отличаются на множестве $C_{T}$ собственных вершинах тупиковых граней. Поэтому для различных комплексов $T$ функции $f_{T}$ являются различными и число таких функций не меньше, чем $\left|R\left(t, T_{0}\right)\right|$. Тогда

$$
\left\{f_{T} \mid T \in R\left(t, T_{0}\right)\right\} \subseteq \mathscr{P}_{n, m, k}(t), \quad\left|R\left(t, T_{0}\right)\right|=\sum_{s \geqslant t z}^{z}\left(\begin{array}{l}
z \\
s
\end{array}\right),
$$

где

$$
z=\left|T_{0} \cup \mathscr{A}\left(T_{0}\right)\right|=2\left|T_{0}\right| \asymp 2^{n} .
$$

Так как

$$
\lim _{z \rightarrow \infty} \frac{1}{z} \log \sum_{s \geqslant t z}^{z}\left(\begin{array}{l}
z \\
s
\end{array}\right)=\hbar(t),
$$

для фиксированного $t$, где $0<t<1$, получаем, что

$$
\log \sum_{s \geqslant t z}^{z}\left(\begin{array}{l}
z \\
s
\end{array}\right) \sim z \hbar(t)
$$

при $z \rightarrow \infty$. Следовательно,

$$
\log \left|R\left(t, T_{0}\right)\right| \sim 2\left|T_{0}\right| \hbar(t) \sim \sigma_{\tau} 2^{n} \hbar(t),
$$

где

$$
\sigma_{\tau}=\varphi(1,0087,0,2104)>0,4025
$$

Перейдем к обоснованию нижней оценки для $p_{n}\left(t, \chi_{\left.\Lambda_{\pi} \cap \Lambda_{l}\right)}\right.$. Для множества функций

$$
P_{n-2, m, k}(t)=t\left\{f \in \mathscr{F}_{m-k, m}^{n-2} \vee \mathscr{F}_{n-2-m, n-2-m+k}^{n-2} \mid \log \tau(f) \gtrsim t c_{\tau} n 2^{n-2}\right\},
$$

где

$$
m=\lfloor(n-2) / 2\rfloor-1, \quad k=\lfloor 0,1052 m\rfloor,
$$

справедлива оценка

$$
\log \left|\mathscr{P}_{n-2, m, k}(t)\right| \gtrsim \sigma_{\tau} \hbar(t) 2^{n-2} .
$$

Обозначим через

$$
\Phi\left[\mathscr{P}_{n-2, m, k}(t)\right]=\left\{\Phi_{f}, f \in \mathscr{P}_{n-2, m, k}(t) \subset P_{n-2}\right\}
$$

подмножество функций $P_{n}$, которые получаются при применении преобразования $\Phi$ к функциям подмножества $\mathscr{P}_{n-2, m, k}(t)$. 
В силу лемм 2-4, для функций из $\Phi\left[\mathscr{P}_{n-2, m, k}(t)\right]$ справедливо неравенство

$$
\log \chi_{\Lambda_{\pi} \cap \Lambda_{l}}(f) \gtrsim t c_{\tau} n 2^{n-2} .
$$

В силу леммы 6 , при применении преобразования $\Phi$ к различным функциям получаются различные функции, то есть

$$
\left|\Phi\left[\mathscr{P}_{n-2, m, k}(t)\right]\right|=\left|\mathscr{P}_{n-2, m, k}(t)\right| .
$$

Поэтому справедлива оценка

$$
\log p_{n}\left(t, \chi_{\Lambda_{\pi} \cap \Lambda_{l}}\right) \geqslant \log \left|\mathscr{P}_{n-2, m, k}(t)\right| \gtrsim \sigma_{\tau} \hbar(t) 2^{n-2} .
$$

Получим нижнюю оценку для $p_{n}\left(t, \tau \mid \mu_{\Lambda_{\pi} \cap \Lambda_{L}}=1\right)$. Для множества функций

$$
\mathscr{P}_{n-3, m, k}(t)=\left\{f \in \mathscr{F}_{m-k, m}^{n-3} \vee \mathscr{F}_{n-3-m, n-3-m+k}^{n-3} \mid \log \tau(f) \gtrsim t c_{\tau} n 2^{n-3}\right\},
$$

где

$$
m=\lfloor(n-3) / 2\rfloor-1, \quad k=\lfloor 0,1052 m\rfloor,
$$

справедлива оценка

$$
\log \left|\mathscr{P}_{n-3, m, k}(t)\right| \gtrsim \sigma_{\tau} \hbar(t) 2^{n-3}
$$

Обозначим через

$$
\Psi\left[\mathscr{P}_{n-3, m, k}(t)\right]=\left\{\Psi_{f}, f \in \mathscr{P}_{n-3, m, k}(t) \subset P_{n-3}\right\}
$$

подмножество функций $P_{n}$, которые получаются при применении преобразования $\Psi$ к функциям подмножества $\mathscr{P}_{n-3, m, k}(t)$.

В силу леммы 5 , для функций из $\Psi\left[\mathscr{P}_{n-3, m, k}(t)\right]$ справедливо неравенство

$$
\log \tau\left(f \mid \mu_{\Lambda_{\pi} \cap \Lambda_{L}}(f)=1\right) \gtrsim t c_{\tau} n 2^{n-3} .
$$

В силу леммы 7, при применении преобразования $\Psi$ к различным функциям получаются различные функции, то есть

$$
\left|\Psi\left[\mathscr{P}_{n-3, m, k}(t)\right]\right|=\left|\mathscr{P}_{n-3, m, k}(t)\right| .
$$

Поэтому справедлива оценка

$$
\log p_{n}\left(t, \tau \mid \mu_{\Lambda_{\pi} \cap \Lambda_{L}}=1\right) \geqslant \log \left|\mathscr{P}_{n-3, m, k}(t)\right| \gtrsim \sigma_{\tau} \hbar(t) 2^{n-3} .
$$

Теорема доказана.

\section{Список литературы}

1. Журавлев Ю. И., Алгоритмы построения минимальных дизъюнктивных нормальных форм для функций алгебры логики. В сб.: Дискретная математика и математические вопросы кибернетики. Наука, Москва, 1974, с. 67-98.

2. Васильев Ю. Л., Глаголев В. В., Метрические свойства дизъюнктивных нормальных форм. В сб.: Дискретная математика и математические вопросы кибернетики. Наука, Москва, 1974, c. 99-148. 
3. Сапоженко А. А., Чухров И. П., Минимизация булевых функций в классе дизъюнктивных нормальных форм. В сб.: Итоги науки и техники. Сер. Теория вероятностей, Математическая статистика, Теоретическая кибернетика. ВИНИТИ АН СССР, Москва, 1987, с. 68-116.

4. Яблонский С. В., Введение в дискретную математику. Высшая школа, Москва, 2003.

5. Журавлев Ю. И., Оценка для числа тупиковых д.н.ф. функций алгебры логики. Сибирский матем. журнал (1962) 3, №5, 802-804.

6. Глаголев В. В., Некоторые оценки д.н.ф. булевых функций алгебры логики. Проблемы кибернетики (1967) 19, 75-94.

7. Сапоженко А. А., О наибольшей длине тупиковой дизьюнктивной нормальной формы у почти всех булевых функций. Матем. заметки (1968) 4, №4, 649-658.

8. Караханян Л. М., Сапоженко А. А., Оценки параметров д.н.ф. не всюду определенных (частичных) функций алгебры логики. В сб.: Комбинаторно-алгебраические методы в прикладной математике, Горький, 1979, с. 48-56.

9. Чухров И. П., Оценка числовых характеристик поясковых функций. В сб.: Тез. докл. V Всесоюзной конф. по проблемам теоретической кибернетики, Новосибирск, 1980, с. 179-180.

10. Чухров И. П., Оценки числа минимальных дизъюнктивных нормальных форм для поясковой функции. Методы дискретного анализа в исследованиях функциональных систем (1981) 36, 74-92.

11. Чухров И. П., О числе тупиковых дизъюнктивных нормальных форм. Доклады АН СССР (1982) 262, №6, 1329-1332.

12. Чухров И. П., О тупиковых комплексах граней в единичном кубе. Дискретная математика (2011) 23, №1, 132-158.

Статья поступила 8.09.2011. 